\title{
Linear Power-Flow Analysis Method FOR AC-DC EleCtric Power Networks
}

by

\section{Mohammadreza Vatani}

Bachelor of Science, University of Tehran, 2010

Master of Science, Amirkabir University of Technology, 2013

\author{
A thesis \\ presented to Ryerson University \\ in partial fulfillment of the \\ requirements for the degree of \\ Master of Applied Science \\ in the program of \\ Electrical and Computer Engineering
}

Toronto, Ontario, Canada 2018

CMohammadreza Vatani, 2018 


\section{AUTHOR'S DECLARATION FOR ELECTRONIC SUBMISSION OF A THESIS}

I hereby declare that I am the sole author of this thesis. This is a true copy of the thesis, including any required final revisions, as accepted by my examiners.

I authorize Ryerson University to lend this thesis to other institutions or individuals for the purpose of scholarly research.

I further authorize Ryerson University to reproduce this thesis by photocopying or by other means, in total or in part, at the request of other institutions or individuals for the purpose of scholarly research.

I understand that my thesis may be made electronically available to the public. 


\title{
Linear Power-Flow Analysis Method for AC-DC Electric Power Networks
}

\author{
Mohammadreza Vatani \\ Master of Applied Science \\ Electrical and Computer Engineering \\ Ryerson University, Toronto, 2018
}

\section{Abstract}

AC-DC power systems have been operating more than sixty years. Nonlinear bus-wise power balance equations provide accurate model of AC-DC power systems. However, optimization tools for planning and operation require linear version, even if approximate, for creating tractable algorithms, considering modern elements such as DERs (distributed energy resources). Hitherto, linear models of only $\mathrm{AC}$ power systems are available, which coincidentally are called DC power flow. To address this drawback, linear bus-wise power balance equations are developed for AC-DC power systems and presented. As a first contribution, while AC and DC lines are represented by susceptance and conductance elements, AC-DC power converters are represented by a proposed linear relationship. As a second contribution, a three-step linear AC-DC power flow method is proposed. The first step solves 
the whole network considering it as a linear AC network, yielding bus phase angles at all busses. The second step computes attributes of the proposed linear model of all AC-DC power converters. The third step solves the linear model of the AC-DC system including converters, yielding bus phase angles at $\mathrm{AC}$ busses and voltage magnitudes at DC busses. The benefit of the proposed linear power flow model of AC-DC power system, while an approximation of the nonlinear model, enables representation of bus-wise power balance of AC-DC systems in complex planning and operational optimization formulations and hence holds the promise of phenomenal progress. The proposed linear AC-DC power systems is tested on numerous IEEE test systems and demonstrated to be fast, reliable, and consistent. 


\section{ACKNOWLEDGEMENTS}

I would like to express my sincere gratitude and heart-felt thanks to Dr. Bala Venkatesh and Dr. Amirnaser Yazdani for their excellent supervision, constant encouragement, continuous aspiration, and kindness throughout this course of work. Without their exceptional guidance and valuable suggestions, this work may not have been completed. I really enjoyed our conversations and discussions. I learned not only technical knowledge but also professionalism and respect. It has been a true privilege to have worked with them, and I am very glad and lucky that I have studied my M.A.Sc degree under their supervision.

I would also like to thank my wife Bahar, for her unconditional support and being by my side during this research. Her spirit, strength, and love encouraged me to overcome the difficulties.

I express my respect and intense gratitude to my mother Mahnaz,and my sister Shakoora who were the source of encouragement and support all through my life and in memory of my father Issa, who has been the source of inspiration and dedication for me. 


\section{DEDICATION}

Dedicated to my beloved wife, Bahar, for her unconditional support,

and my family

Mahnaz and Shakoora 


\section{TABLE OF CONTENTS}

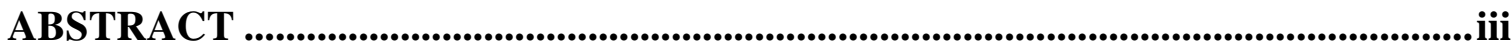

ACKNOWLEDGEMENTS ...................................................................................................... v

DEDICATION

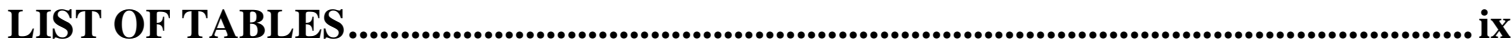

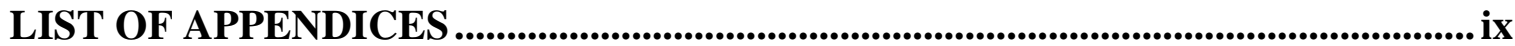

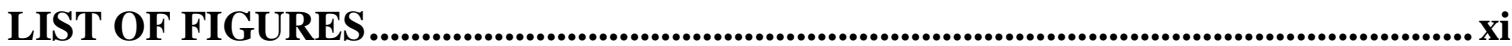

LIST OF ACRONYMS ..............................................................................................xii

LIST OF SYMBOLS .................................................................................................................xiii

INTRODUCTION ........................................................................................................................... 1

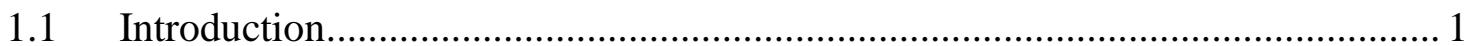

$1.2 \quad$ Literature Survey ...................................................................................... 3

1.2.1 Non-Linear AC-DC Power Flow …………………..................................... 4

1.2.2 Linear AC Power Flow …………............................................................ 7

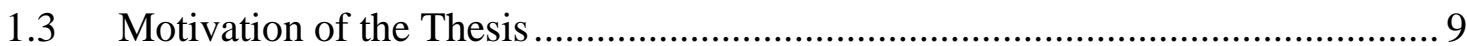

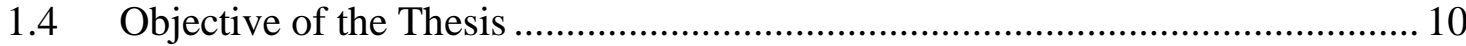

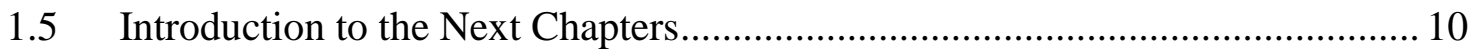

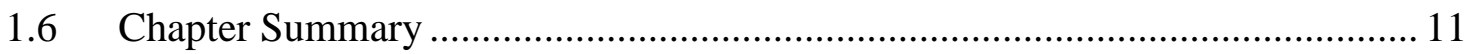

PROBLEM STATEMENT AND PROPOSED SOLUTION ........................................... 12

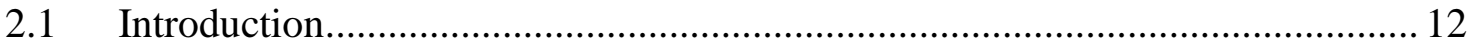

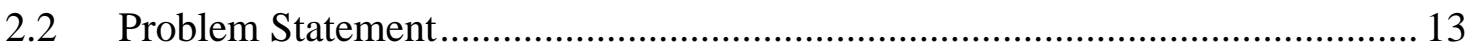

2.2.1 Power Flow of AC Subgrid ......................................................................... 14

2.2.2 Power Flow of DC Subgrid ...................................................................... 17

2.2.3 Linear Power Flow Formulation of AC-DC Networks ..................................... 18

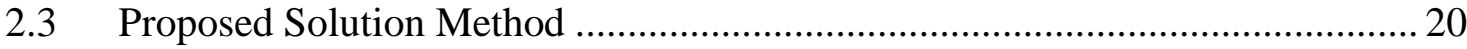




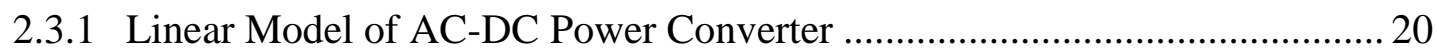

2.3.2 Linear Model for the AC-DC Network ………………………................... 23

2.4 Proposed Three-Step Algorithm ............................................................... 25

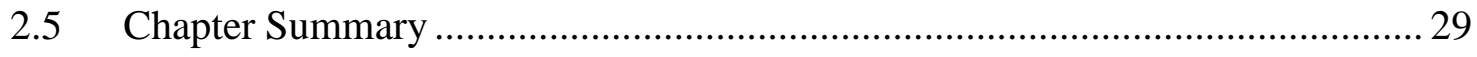

SIMULATION RESULTS.........................................................................................................31

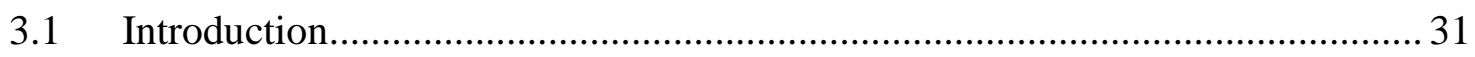

3.2 Numerical Example of 4-Bus Test System...................................................... 33

3.3 Modified IEEE 9-Bus Test System............................................................. 41

3.4 Modified IEEE 14-Bus Test System................................................................. 43

3.5 Modified IEEE 30-Bus Test System............................................................... 47

3.6 Modified New England 39-Bus Test System …………………………........... 50

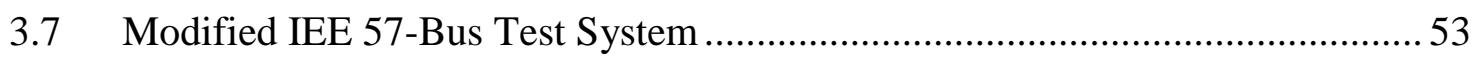

3.8 Modified IEE 118-Bus Test System ............................................................ 55

3.9 Modified IEE 300-Bus Test System ........................................................... 55

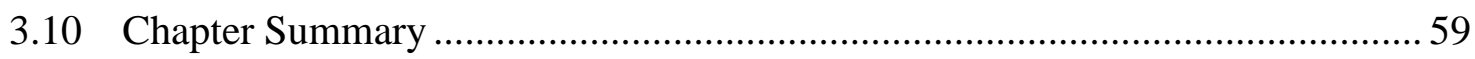

CONCLUSION ........................................................................................................................61

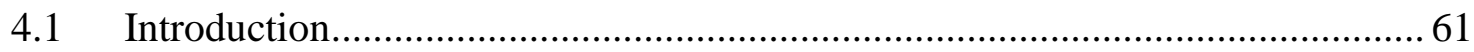

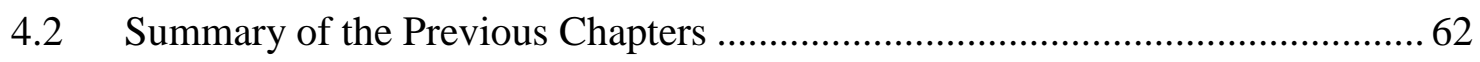

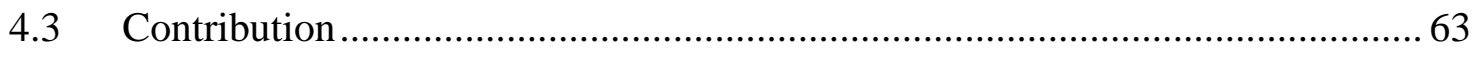

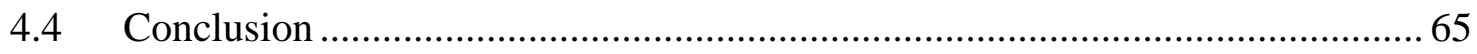

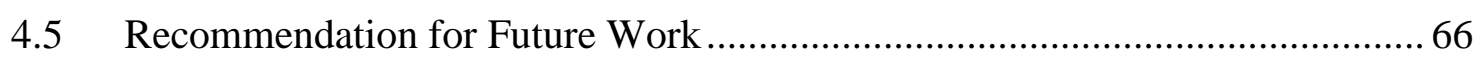

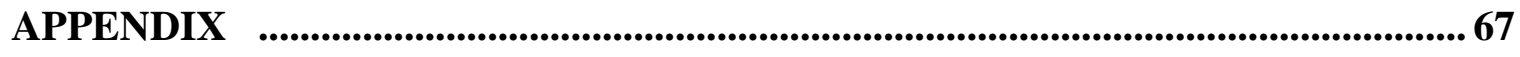

REFERENCES .................................................................................................................. 73 


\section{LIST OF TABLES}

2.1: Comparison of inputs and output of linear power-flow analysis between AC and DC

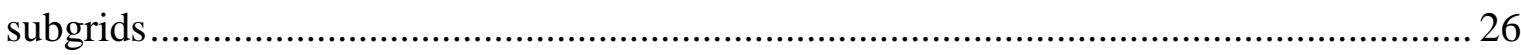

3.1: Summary of the modified test systems ................................................................. 33

3.2: The results of numerical example of 4-bus test system........................................... 40

3.3: Comparison of AC and DC variables results between the proposed linear AC-DC power flow method and the accurate non-linear AC-DC power flow method ........................... 45

3.4: The results of the modified IEEE 30 -bus test system............................................ 49

3.5: The problem of analyzing the results just based on the percent error values ....................50

3.6: The results of the modified IEEE 39 -bus test system............................................ 51

3.7: Summary of the Modified Test Systems ............................................................... 58 


\section{LIST OF APPENDICES}

A.1: Data of the added buses and DC lines to the original IEEE 9-bus test system................ 67

A.2: Data of the added buses and DC lines to the original IEEE 14-bus test system................ 67

A.3: Data of the added buses and DC lines to the original IEEE 30-bus test system............... 68

A.4: Data of the added buses and DC lines to the original IEEE 39-bus test system............... 68

A.5: Data of the added buses and DC lines to the original IEEE 57-bus test system............... 69

A.6: Data of the added AC-DC power converters to the original IEEE 57-bus test system ...... 69

A.7: Data of the added buses and DC lines to the original IEEE 118-bus test system.............. 70

A.8: Data of the added AC-DC power converters to the original IEEE 118-bus test system .... 71

A.9: Data of the added buses to the original IEEE 300-bus test system ............................. 71

A.10: Data of the added DC lines to the original IEEE 300-bus test system ......................... 72

A.11: Data of the added AC-DC power converters to the original IEEE 300-bus test system .. 72 


\section{LIST OF FiguRES}

2.1: Comparison of $x$ and $\sin (x)$ for small angle approximation

2.2: Percent error of small angle approximation 16

2.3: AC-DC power converter model for linear AC-DC power-flow analysis 22

2.4: Flowchart of the proposed method for linear AC-DC power-flow analysis .....................28

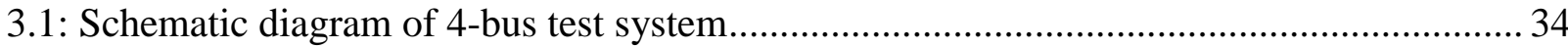

3.2: Equivalent circuit of the 4-bus test system in the third step of the proposed method ......... 37

3.3: Comparison of phase angle error of $\mathrm{AC}$ buses between linear $\mathrm{AC}$ and $\mathrm{AC}-\mathrm{DC}$ power flow methods for the modified IEEE 9-bus test system

3.4: Comparison of power flow error of AC lines between linear AC and AC-DC power flow methods for the modified IEEE 9-bus test system.

3.5: Power flow error of the proposed linear AC-DC power-flow analysis method for different load levels of the modified IEEE 14-bus test system section 46

3.6: Power flow percent error of the modified IEEE 57-bus test system 54

3.7: Mismatch between linear AC-DC and linear AC power flow values in the modified IEEE 57-bus test system

3.8: Percent error of power flow in DC lines and power converters of the modified IEEE 118bus test system 56

3.9: Percent error of power flow in DC lines and power converters of the modified IEEE 300bus test system 


\section{LIST OF ACRONYMS}

$\begin{array}{ll}\text { AC } & \text { Alternative current } \\ \text { DC } & \text { Direct current } \\ \text { HV } & \text { High voltage } \\ \text { MV } & \text { Medium voltage } \\ \text { HVDC } & \text { High voltage direct current } \\ \text { MTDC } & \text { Multi-terminal direct current } \\ \text { VSC } & \text { Voltage-sourced converter } \\ \text { ABS } & \text { Absolute value } \\ \text { DER } & \text { Distributed energy resource }\end{array}$




\section{LIST OF SYMBOLS}

$\mathrm{B}_{\mathrm{ik}} \quad$ Susceptance Matrix in the bus frame of reference, connecting busses $i$ and $k$

[B'] B-prime matrix

[B'Mod] Modified B-prime matrix

[BG] BG matrix

$\mathrm{G}_{\mathrm{ik}} \quad$ Conductance Matrix in the bus frame of reference, connecting busses $i$ and $k$

[MA] Incident matrix of power converters on AC busses

[MD] Incident matrix of power converters on DC busses

nAC Number of buses in AC sub-grid

nDC Number of buses in DC sub-grid

$\mathrm{P}_{\mathrm{i}} \quad$ Active power injection of bus $\mathrm{i}$

$\mathrm{PL}_{\mathrm{NL}} \quad$ Real power flows in lines computed using the accurate nonlinear AC-DC power flow method

$\mathrm{PL}_{\mathrm{Lin}} \quad$ Real power flows in lines computed using the proposed linear AC-DC power flow method

$\mathrm{PL}^{\mathrm{Ave}} \quad$ Average of absolute values of real power flow in lines obtained by the accurate

NL nonlinear AC-DC power flow

$\mathrm{PL}_{\mathrm{Lin}}^{\mathrm{AC}} \quad$ Real power flows in lines computed using the linear AC power flow method

$\left[\mathrm{P}_{\mathrm{AC}}\right] \quad$ Nodal active power injection vector of AC sub-grid

$\left[\mathrm{P}_{\mathrm{DC}}\right] \quad$ Nodal active power injection vector of DC sub-grid

[PCA] Vector of powers flowing into power converters at the AC busses

[PCD] Vector of powers flowing into power converters at the DC busses

T Voltage transfer ratio

$\left[\delta_{\mathrm{AC}}\right] \quad$ Phase angle vector of AC voltages in AC sub-grid

$\left[\mathrm{V}_{\mathrm{DC}}\right] \quad$ Voltage magnitude vector of DC sub-grid

$\mathrm{V}_{\mathrm{i}} \angle \delta_{\mathrm{i}} \quad$ Voltage phasor of bus $\mathrm{i}$

$\mathrm{V}_{\mathrm{x}} \angle \delta_{\mathrm{x}} \quad$ Voltage phasor of hypothetical bus $\mathrm{x}$

$\mathrm{X}_{\mathrm{ik}} \quad$ Reactance of the line between buses $\mathrm{i}$ and $\mathrm{k}$ 
$\mathrm{Xc}_{\mathrm{c}} \quad$ Reactance of the power converter 'c'

$\lambda$ Parameter relating the AC and DC variables of an ideal converter

$\mathrm{i}, \mathrm{k} \quad$ Indices for buses 


\section{ChAPTER 1.}

\section{INTRODUCTION}

\subsection{Introduction}

AC-DC power systems have operated well over a half a century. Optimal planning and operations for AC-DC large-scale power systems are very important. These mixed integer optimization formulations include expansion planning for transmission systems, unit commitment and optimal power flow. These formulation model AC-DC transmission system elements using a set of bus-wise power balance equations, which are nonlinear and nonconvex.

In several instances, considering large-scale power AC power systems, transmission system is usually modelled using a linear equivalent model, called DC power flow equations. It 
makes these large scale problems more tractable. However, linear model of AC-DC power systems does not exist and hence their optimization formulations for planning and operations remain a computational challenge.

Due to the recent advances in energy storage devices and techniques, DC cables, electric vehicles, and AC-DC power converters, more DC devices are interfaced with the legacy power system. DC networks exhibit advantages over AC ones in terms of voltage regulation, power losses, power quality, reliability, and more straightforward accommodation of renewable energy resources [1]. In this regard, lately, the attention toward medium-voltage (MV) DC microgrids has increased among researchers. Thus, the future power system is expected to have AC-DC architecture in which loads and generation in both AC and DC forms with different sizes and voltage levels co-exist [2]. For instance, the first MV grid-connected battery energy storage system was commissioned in 2016 [3]. City infeed [4] and the connection of offshore wind farms to power systems [5] are two other examples of MV AC-DC networks.

In order to have a successful transition from the legacy AC power system to an AC-DC power system, some existing analysis tools should be modified while some new ones are to be produced. The power-flow analysis is one such a basic tool for power system simulation, analysis, and design. Non-linear AC-DC power flow is currently being developed through research studies. However, there is a strong need for a linear power-flow analysis method for AC-DC electrical networks.

Power-flow analysis is required for almost any power system project from establishing a new power plant or transmission line to optimally manage the generation of generators for loss reduction purposes. So much significant is the role of power-flow analysis in industry, 
electrical utilities, and academia that different power systems analysis software companies have spent noticeable efforts to develop and increase the efficiency and accuracy of their power-flow analysis toolbox. They should keep pace with new technologies, configurations, and operations which are applied to power systems.

Considering the above explanation about the importance of power flow tools in power system study, planning, and operation; this thesis intends to solve the problem of linear AC-DC power-flow analysis in AC-DC electrical networks. The linear power-flow analysis has a wide range of applications in power system and energy markets. However, to the best of the author's knowledge, there is still no reliable and straightforward method as a solution to this problem.

\subsection{Literature Survey}

The operation of AC-DC electrical networks requires more knowledge about its capabilities and possible configurations. In $[6,7]$, the main benefits of AC-DC networks are pointed. For instance, it makes it easier and more efficient to integrate the AC- or DC-based devices (e.g., loads, generators, energy storage, and electric vehicles) to the network because it minimizes the need for interface devices and reduces the conversion stages. Moreover, voltage transformation and support can be achieved in DC subgrids by the use of DC-DC converters without use AC transformers. However, there are some significant challenges for AC-DC networks like reliability, protection and control complexity.

The recent works related to the title of this thesis are briefly introduced and reviewed in this section. They are classified into three different topics including AC-DC electrical networks, non-linear AC-DC power flow, and linear AC power flow. Having a general 
overview of AC-DC network, its applications, configurations, and definitions is essential. Moreover, surveying the recent works about non-linear AC-DC power flow gives us the ideas and models based on which the new linear methods and algorithms may be established. Finally, reviewing the linear $\mathrm{AC}$ power-flow analysis method enables us to be familiar with the approximations and assumptions which should be made for linearizing the load models and power flow equations.

\subsubsection{Non-Linear AC-DC Power Flow}

Different sequential and unified non-linear techniques have been proposed for AC-DC power-flow analysis for distribution systems, industrial networks, MV microgrids, and highvoltage direct-current (HVDC) transmission systems. In [2], a unified approach for AC-DC power-flow analysis in islanded microgrids is proposed. AC and DC subgrids, their components, and their AC-DC power converters are mathematically modelled. The linear and non-linear equations are then solved simultaneously by using a globally convergent Newton trust-region method. Moreover, some special conditions of an islanded AC-DC microgrid such as the bidirectionality of the power flow and the droop-based control systems of converterbased distributed generations are considered.

An improved method for AC-DC power-flow analysis with multi-infeed DC systems is proposed in [8]. This method enhances the performance and the accuracy of calculation by avoiding unfeasible solutions and interruptions when AC-DC power flow is calculated. Three suggestions are introduced to improve convergence properties and speed of AC-DC power flow calculation by adjusting the AC-DC power converter transformer tap positions. The burden of AC-DC power flow calculation is noticeably reduced by calculating each DC system as an 
individual calculation unit and independent to other DC systems. A generalized steady-state model for the multi-terminal voltage-sourced converter (VSC) with is introduced in [9]. The converter equations are derived with further elaborations on converter step-up transformer, filter, and operation limits. The introduced model can be used in sequential AC-DC power-flow analysis algorithms. Additionally, connection of multiple DC subgrids within one AC network is considered in this model.

A unified power flow algorithm based on Newton-Raphson method with a detailed model of the VSC including the converter power losses is presented in [10]. A model of DC subgrid is constructed which is compatible with different DC voltage control modes and any AC-DC networks topologies. Three separate sets of mismatch equations and unknown variables corresponding to AC, VSC, and DC models are defined. Finally, the quadratic convergence of the algorithm is obtained by using the Newton-Raphson method. A $\pi$-model of controllable AC-DC converters is proposed in [11]. This model can be applied even to unbalanced threephase systems in which the AC-DC power converters are controlled through current controllers. Independent to the control strategy of the AC-DC power converter, it is modelled as an impedance in series with an ideal AC-DC converter.

A unified admittance matrix can be obtained for the entire AC-DC system by using the $\pi$ model of the AC-DC controllable converter proposed in [11]. Then, the Newton-Raphson power-flow analysis method can be utilized by having an admittance matrix for the overall ACDC network. A method for AC-DC power flow studies in industrial AC-DC power systems is suggested in [12]. Special features of certain industrial AC-DC distribution systems such as large commutation resistances as well as the approximation of a fixed-magnitude relation 
between AC and DC currents of an AC-DC converter are taken into account in this paper. The effects of the aforementioned parameters on system power flow are indicated by deriving a detailed model of the converter for use in a Newton-Raphson power flow program.

A unified power flow model for AC-DC distribution systems is proposed in [13] which introduces a new classification of the system buses for power flow analysis. In this paper, the AC-DC distribution system is based on VSCs. Possible topologies of AC-DC distribution systems are analyzed and described by three binary matrices. These matrices are utilized to form the unified power equations allowing the method to have a high degree of flexibility. They identify the type of the configuration in power equations at a certain time. This method is applicable to both grid-connected and islanded operation modes of distribution systems. Another unified power flow algorithm for AC-DC power systems is presented in [14]. The ACDC converter is modelled by the minimum combination of state variables which are voltage magnitude and phase angle of the AC node and the voltage magnitude of the DC node of the AC-DC converter. This decreases the complexity of the unified power flow algorithm. The aforementioned state variables are defined and calculated under diverse control strategies of VSC. Then, the bus-power-equilibrium equations of AC and DC nodes of VSC are modified for the unified power flow algorithm.

In [15], A new algorithm for power-flow analysis for AC-DC power systems with offshore wind farms is proposed. The AC mismatch equations are updated by the transferred power at the AC-DC converter-interfacing buses. The modulation index control of the AC-DC converter is modelled. The proposed algorithm in this paper is suitable to be implemented for optimal power flow analysis, load flow congestion studies, unity dispatch and static security 
assessment problems of hybrid networks. A new power flow calculation approach for AC-DC grids including VSC-based multi-terminal DC networks is introduced in [16]. The converter losses are considered in this approach while it can be categorized as a unified AC-DC power flow approach. The AC-DC converter losses and control modes are handled by introducing a new state variable. The method is built upon a unit called general unified AC-DC unit. Then, two different analysis methods are introduced and compared based on modification requirements and computational time. Another AC-DC power flow model with VSC multiterminal HVDC is suggested in [17]. The proposed method consists of two nodal equations and two power constraints for each $\mathrm{AC}$ bus and one nodal equation and one power constraint for each DC bus. The VSC equation is split into the AC and DC network model which decreases the total number of equations for the complete AC-DC network and consequently enhances the computational efficiency. The method is independent of VSC control strategy and configuration.

\subsubsection{Linear AC Power Flow}

Linear power-flow analysis plays a significant role in power system planning and operation. It is widely used for contingency analysis [18, 19], real-time security-constrained economic dispatch [20], unit commitment [21], and medium-to-long term transmission expansion planning [22]. The aim of linear power-flow analysis is to calculate the amount of active power passing through each transmission lines. Various approximations and assumptions have been proposed for linearizing the AC power balance equations or load models [23, 24]. The impact of these assumptions on the accuracy of the linear power flow results is studied in [23]. 
In [25], a new load model is introduced which considers the load voltage dependency. The load is divided in this paper into an impedance and a current source based on a simple curve-fitting technique. This model can be used for linear power flow analysis with some numerical approximations on the imaginary parts of the nodal voltages. The method in [25] is modified in [26] to meet the load unbalance conditions of distribution systems. Assuming the negligible voltage angles and magnitudes variations in distribution systems, a three-phase linear power flow formulation is presented. In [24], another linear power flow method for unbalanced and balanced three-phase power distribution systems is suggested which considers the ZIP models of the loads. This method is appropriate for the networks with high $R / X$ ratio. Another linear power flow method for unbalanced active distribution network is proposed in [27]. PV nodes and ZIP model of the loads are considered in this paper. Probabilistic power flow, convex optimal power flow, and volt-VAR control are good applications of this method. In [28], a linear power flow technique for power system reliability studies is introduced. This method provides the approximate values of both bus voltage magnitudes and phase angles. The proposed method is suitable to be used for those test systems with less than 118 buses. The linear AC power-flow analysis is modified in [29, 30] for phase shifting transformer applications in power system and optimal power-flow analysis. In [31], a new linear active power flow is introduced. This method is based on decoupling principles solving phase angle and magnitude of the voltage in decoupling form. Moreover, the aforesaid method takes the resistance of transmission lines into the account. It means that it does not assume the high value of $X / R$ ratio. However, the procedure of the proposed method is not as simple as the classical linear AC power-flow analysis method. 
As the summary of literature survey, it can be said that AC-DC power systems have gained researchers' attention in diverse fields such as configurations, control strategy, energy management, and power electronics. As a power system study point of view, power-flow analysis methods should be available for planning and operation of AC-DC electrical networks. Although different unified and sequential non-linear AC-DC power-flow analysis methods have recently proposed or developed for different types of applications, to the best of the author's knowledge, no linear AC-DC power-flow analysis method can be found in the recent literature and a new method is required.

\subsection{Motivation of the Thesis}

Nowadays; due to the existence of energy market, real-time pricing, different contingency analysis methods, and etc.; the operation of power systems has become more dependent upon fast power system analysis toolboxes like linear power flow. Moreover, the recent advancement of technologies has enabled us to utilize the high-voltage high-power semiconductor switches and devices increasing the efficiency of power systems through its operation in AC-DC configuration. However, technical tools and methods are required to pave the way toward the aforementioned purpose. One of these important tools is linear power-flow analysis. Although different methods for non-linear AC-DC power-flow analysis are suggested, to the best of the authors' knowledge, the linear AC-DC power flow is not studied. Therefore, it was a motivation to fill the gap among the researches related to AC-DC power-flow analysis. 


\subsection{Objective of the Thesis}

In this thesis, the main focus is to provide a linear power-flow analysis method for ACDC electrical networks. The method is based on finding a matrix representing the entire ACDC network including AC subgrid, DC subgrid, and AC-DC power converter. Another focus is to find a linear model of AC-DC power converter to be incorporated in the aforementioned matrix. Finally, a straightforward procedure of the proposed method is presented.

\subsection{Introduction to the Next Chapters}

In Chapter 2, a general overview of the problem and require background are stated. Then, the problem is mathematically defined and the general form of the solution is formulated. The assumptions which should be taken in account for solving the problem are introduced. After that, the proposed method is described in detail as a solution for the defined problem. The proposed linear model of an AC-DC power converter is demonstrated. Then, the incorporation of this model into the AC-DC networks is explained. Finally, the procedure of the proposed linear AC-DC power-flow analysis method is presented and the flowchart of the procedure is illustrated.

Chapter 3 is devoted to reporting the simulation results of the proposed method. First, a numerical example of a 4-bus test system is presented. The step-by-step procedure of the proposed method is elaborated on this test system. MATLAB/Simulink software is utilized to simulate the 4-bus test system. The proposed method is programmed in MATLAB software. Moreover, several test systems of different sizes are used to confirm the performance of the proposed method. Different indices are defined to comprehensively present the results. The 
results of the proposed method are compared with a non-linear AC-DC power-flow analysis method using $\pi$-model of AC-DC controllable converters [11]. The $\pi$-model of the AC-DC converters is also programmed in MATLAB software. The accuracy and the performance of the proposed method are discussed for each test system.

In Chapter 4, the conclusion of each chapter is expressed. Then, the major contributions of the research work are described and the main conclusion of the entire work is given. Finally, some recommendations are suggested for future works.

\subsection{Chapter Summary}

In this chapter, the topic of this thesis is introduced. The industrial and academic relevance of the topic is described. Then, the problem which is intended to solve and the importance of its solution for electric utilities are presented. The recent researches on this topic are studied. The literature review points the main contributions and drawbacks of the recent work and helps to find the gap among them. Then, the motivation and objective of the thesis are mentioned. Finally, the introduction to the titles of the next chapters and their contents are given. 


\section{CHAPTER 2.}

\section{Problem Statement AND}

\section{Proposed Solution}

\subsection{Introduction}

This chapter is devoted to stating the problem associated with the linear power-flow analysis in AC-DC electric power networks as well as to proposing a method for solving the introduced problem. Different linear power-flow analysis methods for AC and DC networks are already utilized in diverse power system analysis software and also in academic research. Hence, the missing part is a linear model of AC-DC power converters to be joint to the linear models and equations of AC and DC subgrids. In this regard, a review of linear power-flow 
analysis methods for both AC and DC subgrids are presented. The related assumptions, equations, and procedure are also expressed since they may be referred to or used in the next parts. Then, the general form of linear AC-DC power-flow equation is conceptually obtained based on the linear power-flow formulations of AC and DC subgrids.

The aim of this chapter is to propose a method for linear AC-DC power-flow analysis. As the first step, a linear model of AC-DC power converter is introduced and then, the linear active powers bidirectionality flowing through the AC-DC power converter are achieved. These two linear equations represent the linear active power flowing through AC-DC power converter from the AC side to DC side and vice versa. The linear power flow equations of AC-DC power converter are formulated in nodal injection form when AC and DC subgrids are connected to the AC-DC power converter. Consequently, a matrix which is referred in this thesis to as $[\boldsymbol{B G}]$ matrix is introduced which represents the entire AC-DC network.

The procedure of the proposed linear AC-DC power-flow analysis method is explained in the last part of this chapter. The procedure includes three steps. The aim of the first and the second steps is to find the parameter $\lambda$. This parameter should be determined to make us able to establish the $[\boldsymbol{B} \boldsymbol{G}]$ matrix. Once the value of $\lambda$ is calculated, the $[\boldsymbol{B} \boldsymbol{G}]$ matrix can be formed and the linear AC-DC power-flow equation can be solved. Then, the unknown variables of AC and DC subgrids can be obtained.

\subsection{Problem Statement}

An AC-DC network consists of an AC subgrid, a DC subgrid, and an AC-DC power converter which interlink those subgrids. The linear power-flows equations for AC and DC 
subgrids are presented first in this section. Then, the general form of linear power-flow equations for the overall AC-DC network is presented by taking the AC-DC power converter into the account. The general form of linear AC-DC power-flow equation is defined based on a matrix representing the AC-DC network entirely. This matrix is called $[\boldsymbol{B G}]$ matrix in this thesis.

\subsubsection{Power Flow of AC Subgrid}

The active power injection of an $\mathrm{AC}$ bus, $i$ in an $\mathrm{AC}$ sub-grid is given by:

$$
P_{i}=V_{i} \sum_{k \in n A C} V_{k} \cdot\left(G_{i k} \cos \delta_{i}-\delta_{k}+B_{i k} \sin \delta_{i}-\delta_{k}\right)+\sum_{c \in i} P C A_{c} \quad \forall i \in n A C
$$

where $P C A_{c}$ is the value of power flowing into power converter at the AC bus, $i$.

The aim of the linear power flow is to find the active power flowing through each line. To linearize (2.1), the following assumptions were adopted as those presented in [32] should be taken into account.

Assumption \#1: The ratio of line reactance to resistance is assumed to be high, typically

greater than 4 (i.e., $\frac{x}{r} \geq 4$ ). The value of four (4) limits the error caused by this assumption [23] and can be found in lines with voltages equal to or higher than $25 \mathrm{kV}$.

The admittance of each line can be represented by the following equation:

$$
\left[B^{\prime}\right]=\left.[B]\right|_{\text {resistance }=0}
$$


Assumption \#2: In a healthy power system, the voltage magnitudes of the buses, in per unit, are close to unity and hence assumed to be unity. In practice, the voltage magnitudes are in a range between 0.95 p.u to 1.05 p.u.

Assumption \#3: It is also assumed that the phase angles of voltage phasors are close to each other and, therefore, the following approximation can be made, when expressed in radians:

$$
\sin \left(\delta_{i}-\delta_{k}\right) \approx \delta_{i}-\delta_{k}
$$

In order to validate the given approximation in (2.3), the comparison between the angle value and its sinusoidal function is shown in Figure 2.1. The angle is represented in degree and the output function of $x$ is expressed in radian. This figure indicates that the value of $\sin (x)$ is very close to $x$ for small values of angle. The difference value between these two functions increases when the angle value gets larger. The error value between $\sin (x)$ and $x$ as a function of angle is illustrated in Figure 2.2. It can be observed that the percent error value is negligible when the angle is less than 5 degrees. However, the error value can be limited to $5 \%$ if the angle $x$ is less than 30 degrees. 


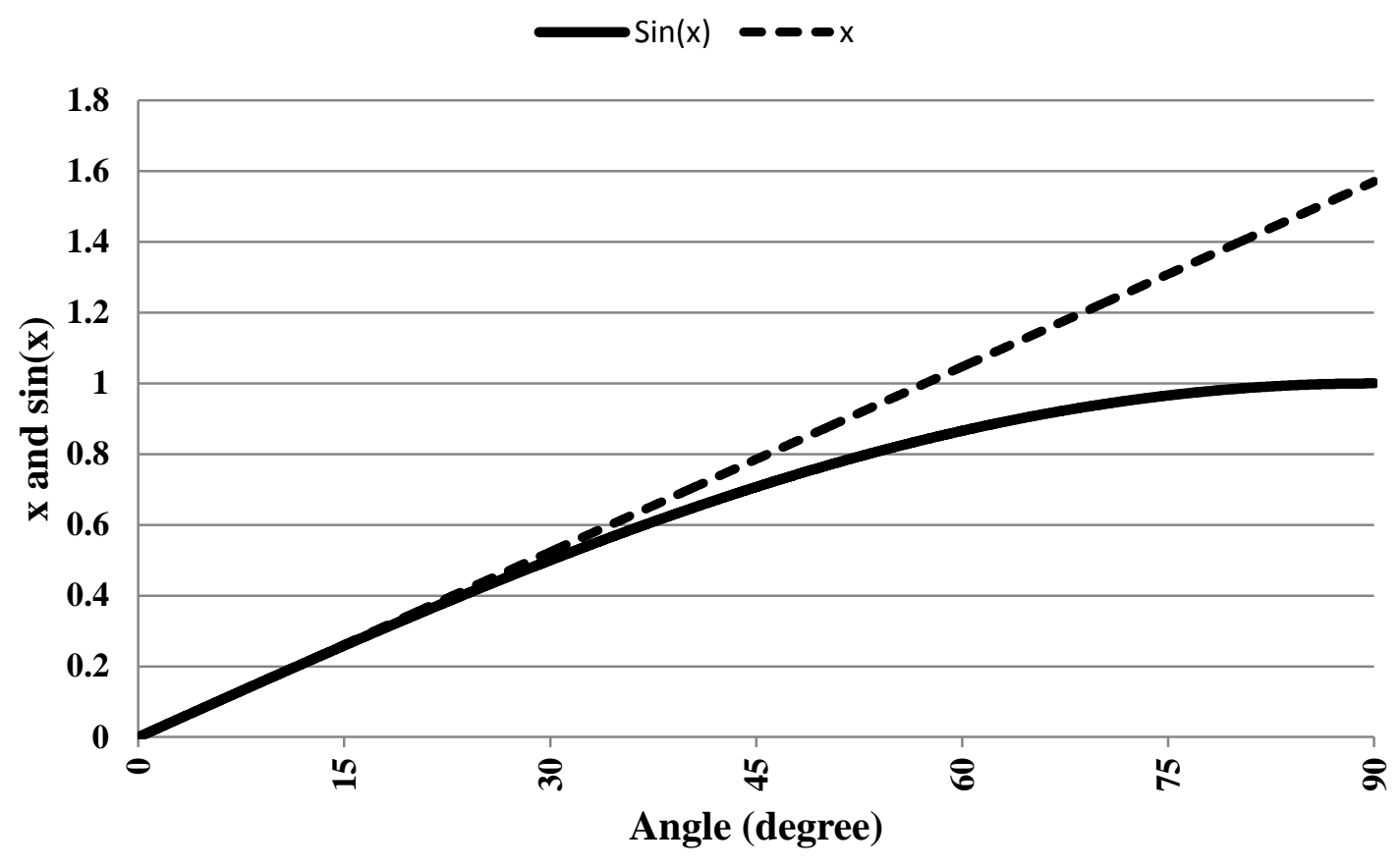

Figure 2.1: Comparison of $x$ and $\sin (x)$ for small angle approximation

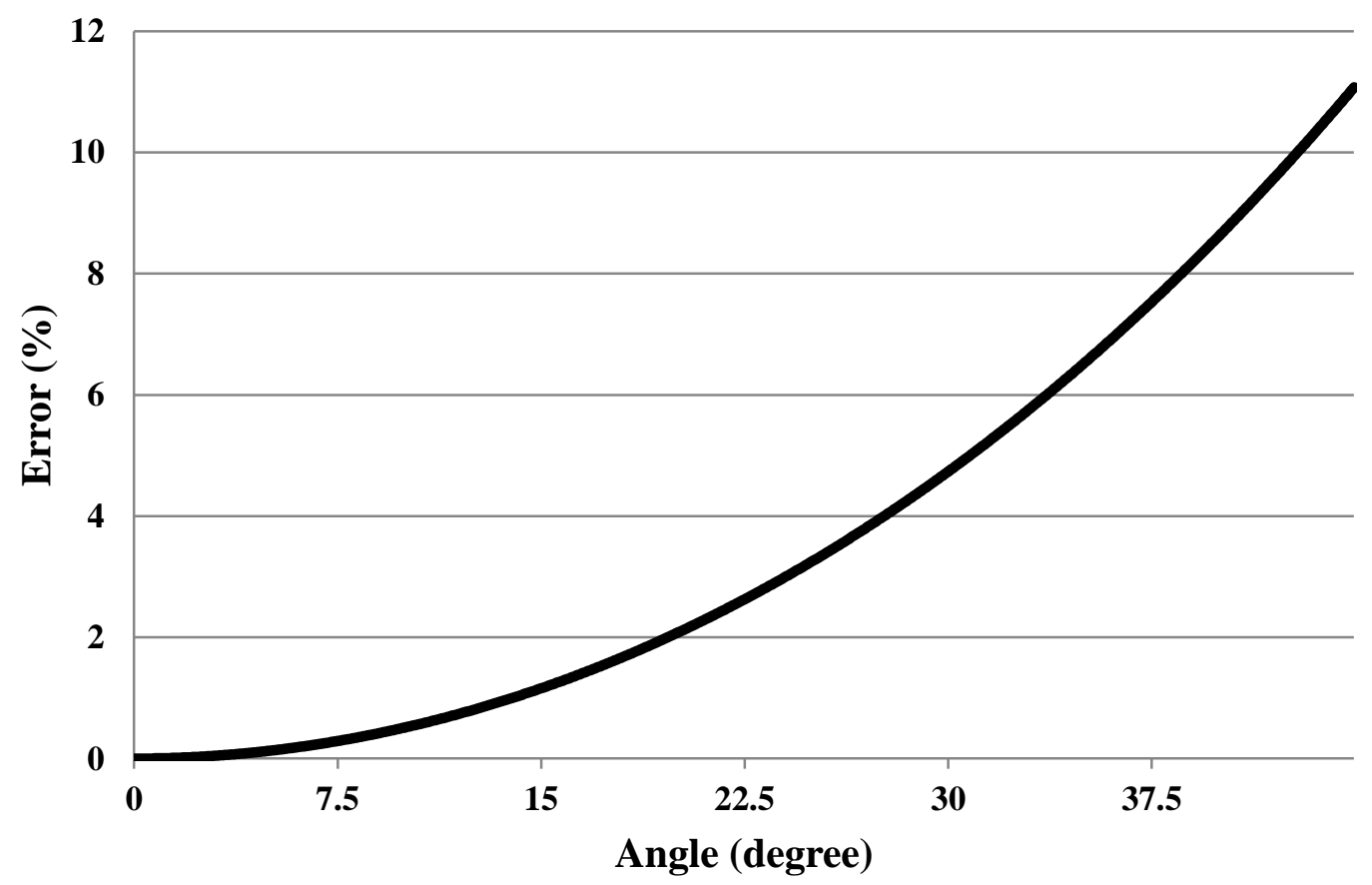

Figure 2.2: Percent error of small angle approximation 
In most normal operating conditions, $\delta_{i k}$ is smaller than 30 degrees ensuring a low value of error caused by this assumption.

Based on the aforementioned assumptions, the linear form of (2.1) is given by

$$
P_{i}=\sum_{k \in n A C} B_{i k} \cdot \delta_{k}+\sum_{c \in i} P C A_{c} \quad \forall i \in n A C
$$

which can be presented in a matrix form as

$$
\left[P_{A C}\right]=\left[B^{\prime}\right]\left[\delta_{A C}\right]+[M A][P C A]
$$

where $\left[B^{\prime}\right]$ is B-prime matrix. $\left[P_{A C}\right]$ and $[\delta]$ are vectors of bus-wise active power injections and phase angle of voltage phasors, respectively. $[M A]$ and $[P C A]$ are incident matrix of power converters on $\mathrm{AC}$ busses and vector of powers flowing into power converters at the $\mathrm{AC}$ busses. Matrix $[M A]$ is defined as below:

$$
M A_{i c}=\left\{\begin{array}{cc}
1 & \text { if power converter c connects } A C \text { bus } i \\
0 & \text { otherwise }
\end{array}\right.
$$

\subsubsection{Power Flow of DC Subgrid}

The transmission lines in a DC subgrid are purely resistive. Moreover, the voltages and powers are real numbers. Thus, the power injection of a DC bus $i$, in a DC subgrid is given by:

$$
P_{i}=V_{i} \cdot I_{i}=V_{i} \sum_{k \in n D C} G_{i k} \cdot V_{k}+\sum_{c \in i} P C D_{c} \quad \forall i \in n D C
$$


where $G_{i k}$ is the element of conductance matrix positioned at $i$ th row and $k$ th column.. The product of the voltages causes a non-linearity in (2.7). However, if $V_{i}$ is assumed to be equal to 1 p.u., the following linear equation approximates the power flow of the DC network:

$$
P_{i}=\sum_{k \in n D C} G_{i k} \cdot V_{k}+\sum_{c \in i} P C D_{c} \quad \forall i \in n D C
$$

Equation (2.8) can be written in a matrix form as:

$$
\left[P_{D C}\right]=[G]\left[V_{D C}\right]+[M D][P C D]
$$

where $[G]$ is conductance matrix of the DC sub-grid and $\left[P_{D C}\right]$ and $\left[V_{D C}\right]$ denote the nodal active power injection vector and nodal voltage vector in DC subgrid, respectively. $[M D]$ and $[P C D]$ are incident matrix of power converters on DC busses and vector of powers flowing into power converters at the DC busses. Matrix $[M D]$ is defined as below:

$$
M D_{i c}=\left\{\begin{array}{lc}
1 & \text { if power converter } c \text { connects } D C \text { bus } i \\
0 & \text { otherwise }
\end{array}\right.
$$

\subsubsection{Linear Power Flow Formulation of AC-DC Networks}

Equations (2.5) and (2.9) give the matrix forms of linear power-flow equations for the AC and DC subgrids. There also exists an AC-DC power converter that interlinks these AC and DC subgrids. The AC-DC power converter has an AC bus and one DC bus. Any types of AC loads and generations may be connected to the AC side of the AC-DC power converter. The DC loads and generators also may be connected to the DC side of the converter. Therefore, the DC and $\mathrm{AC}$ sides of this converter must be included in the nodal power injection vector. Similarly, two more variables are included in the vector of unknown variables. The corresponding 
unknown variable to the $\mathrm{AC}$ side of the AC-DC power converter is the phase angle of the voltage and the corresponding unknown variable to the DC side of the AC side of the AC-DC power converter is the magnitude of the voltage.

Like the B-prime matrix of the AC subgrids and the G-prime matrix of DC subgrids, a matrix should be defined here to relate the nodal active power injection vector to the unknown variables of the AC-DC network and also to presents the entire AC-DC network in just one matrix. This matrix is referred in this thesis to as $[\boldsymbol{B G}]$ matrix. It is called BG since it has the same function as $\left[B^{\prime}\right]$ and $\left[G^{\prime}\right]$ in the AC and DC subgrids, respectively and it also includes both of them. The linear equations for AC-DC power flow can be expressed in a matrix form as:

$$
\left[\begin{array}{l}
\boldsymbol{P}_{A C} \\
\boldsymbol{P}_{D C}
\end{array}\right]=[B G]\left[\begin{array}{l}
\boldsymbol{\delta}_{A C} \\
\boldsymbol{V}_{D C}
\end{array}\right]
$$

in which $P_{A C}$ and $P_{D C}$ are the nodal power injections of $\mathrm{AC}$ and DC subgrids including the nodal power injections of AC and DC sides of the AC-DC power converter, respectively. Moreover, $\delta_{A C}$ and $V_{D C}$ are the unknown variables of $\mathrm{AC}$ and DC subgrids including the phase angle of AC bus voltage and voltage magnitude of DC bus of the AC-DC power converter, respectively. However, the nodal power injection of AC-DC power converter buses must be expressed in terms of its unknown variables. These relationships are established in Section 2.3.2. Equation (2.11) has the same appearance of linear AC power-flow analysis method in which the unknown variables are related to the nodal active power injection vector through a matrix. This matrix can represent the entire AC-DC network in linear power-flow analysis. Hence, we should look for a matrix which is called here as $[\boldsymbol{B} \boldsymbol{G}]$ matrix. The AC and DC subgrids in an AC-DC network are represented by their corresponding matrices $\left[B^{\prime}\right]$ and $\left[G^{\prime}\right]$, 
respectively. Therefore, we should find a way to incorporate the AC-DC power converter model in $[\boldsymbol{B} \boldsymbol{G}]$ matrix.

\subsection{Proposed Solution Method}

In this section, the proposed method for AC-DC power-flow analysis is presented. The proposed method is based on the linear model of AC-DC power converters. Therefore, in the first part of this section, the proposed linear model of the AC-DC power converter is introduced. Then, the inclusion of the linear model of the converter to a typical AC-DC network is given. This is an essential step since we aim to achieve the linear model for the entire AC-DC network. Finally, the procedure of the proposed method for linear AC-DC power flow analysis is described. The procedure consists of three steps which make a straightforward process for obtaining the results.

\subsubsection{Linear Model of AC-DC Power Converter}

The proposed method in this study is limited to the steady state operation of the AC-DC power converters. Moreover, it is assumed that the converter is lossless. Therefore, the active power is balanced between the AC and DC sides of the AC-DC power converter. Consider the $c^{\text {th }}$ AC-DC power converter connecting the $i^{\text {th }}$ bus of the AC sub-grid and $k^{\text {th }}$ bus of the DC sub-grid. The unknown variables of the AC-DC power converter for linear power flow analysis are the phase angle of the AC bus voltage $\delta_{i}$ and the magnitude of the DC bus voltage $V_{k}$. The aim of the converter model for linear power-flow analysis is to describe the active power at both sides of the converter in terms of the aforementioned unknown variables. 
Equations (5) and (9) give the matrix forms of linear power-flow equations for the $\mathrm{AC}$ and DC sub-grids. However, there also exist AC-DC power converters that interlink these subgrids and terms $[P C A]$ and $[P C D]$ require a linear framework. Therefore, for each power converter, an internal fictitious AC bus is created with a state $V_{x} \angle \delta_{x}$. The load at this bus equals zero. For the purpose of this representation, $V_{x}=1$ p.u.

The schematic diagram shown in Figure 2.3 is used to derive the AC-DC power converter model for linear AC-DC power-flow analysis. The diagram consists of two components: the reactance $x c_{c}$ and the voltage conversion block represented by an ideal AC-DC power converter, respectively. They account for the equivalent reactance and the voltage transformation of the converter, respectively [11, 33]. A fictitious bus labelled as $x$ is introduced to connect these two elements of Figure 2.3 As shown in this figure, the voltage magnitude of $\mathrm{AC}$ bus voltages, including the fictitious bus $x$, is equal to 1 p.u. based on the second assumption in Section 2.1. It is worth mentioning here that the fictitious bus $x$ is considered as an AC bus since it is connected to the AC side of the ideal AC-DC converter. Hence, it has a voltage magnitude equal to 1 p.u. and a phase angle of the voltage defined as $\delta_{x}$. This variable will be utilized to relate the AC bus of the AC-DC power converter to its DC bus and thereafter, $\delta_{x}$ will be eliminated from the equations 


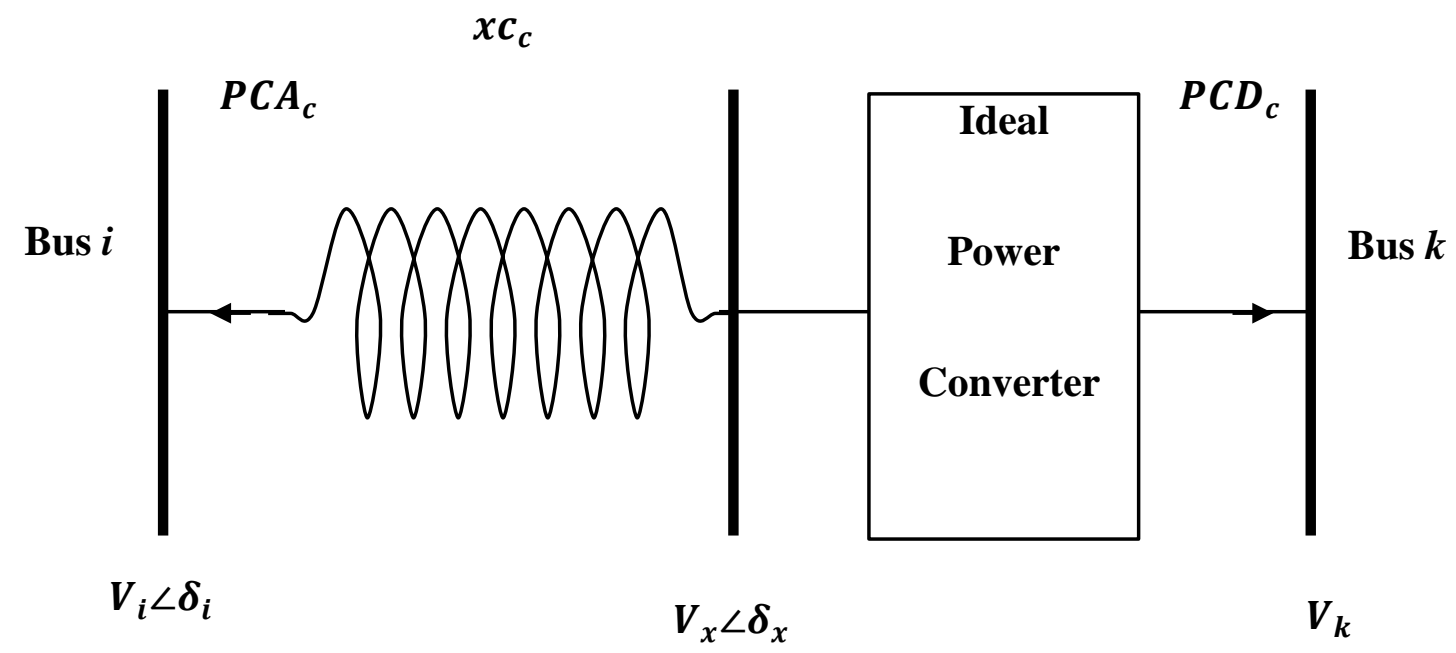

Figure 2.3: AC-DC power converter model for linear AC-DC power-flow analysis

Assume the following relationship between the variables of the AC and DC sides of the ideal AC-DC converter (i.e., $\delta_{x}$ and $V_{k s}$ ):

$$
\delta_{x}=\lambda V_{k}
$$

The procedure of finding the value of $\lambda$ will be presented later.

Based on the equations for linear $\mathrm{AC}$ power flow, the power transferred from $\mathrm{AC}$ to DCside of the AC-DC power converter is

$$
P C A_{C}=\frac{\delta_{i}-\delta_{x}}{x c_{c}}
$$

Substituting from (2.12) to (2.13) produces: 


$$
P C A_{C}=\frac{1}{x c_{c}} \delta_{i}-\frac{\lambda_{c}}{x c_{c}} V_{k}
$$

Equation (2.14) presents the active power flowing from $\mathrm{AC}$ side of the $\mathrm{AC}-\mathrm{DC}$ power converter to its DC side.

In the matrix form, (2.14) is as below:

$$
P C A=[d(x c)]^{-1} \cdot[M A]^{t} \cdot \delta-[d(\lambda)] \cdot[d(x c)]^{-1} \cdot[M D]^{t} \cdot V
$$

Assuming a lossless AC-DC power converter, the following equations can be written:

$$
\begin{gathered}
P C A_{c}+P C D_{c}=0 \\
P C D_{c}=-\frac{1}{x c_{c}} \delta_{i}+\frac{\lambda_{c}}{x c_{c}} V_{k}
\end{gathered}
$$

Equation (2.17) expresses the active power flowing from the DC side of the AC-DC power converter to its $\mathrm{AC}$ side. It can be written in the matrix form as:

$$
P C D=-[d(x c)]^{-1} \cdot[M A]^{t} \cdot \delta+[d(\lambda)] \cdot[d(x c)]^{-1} \cdot[M D]^{t} \cdot V
$$

Equations (2.15) and (2.18) are the basic materials for establishing the BG-prime matrix and the general form of linear AC-DC power-flow analysis as presented in (2.11).

\subsubsection{Linear Model for the AC-DC Network}

Equations (2.15) and (2.18) are linear equations of active power flowing through the ACDC power converter from AC side to DC side and vice versa. However, the aim of this thesis is to propose a method for linear AC-DC power-flow analysis the general form of which is presented in (2.11). It should be noted that the proposed method is independent of number of AC-DC power converters in the AC-DC network. In general, the AC-DC power converter can 
be connected to any number of buses of AC or DC subgrids. Moreover, one or more AC or DC subgrids can be connected to the AC-DC power converter. Each AC or DC subgrid might have a radial or interconnected configuration.

Equations (2.15) and (2.18) are used in Equations (2.5) and (2.9) to create a linear model of the entire AC-DC power system as below:

$$
\begin{gathered}
{\left[P_{A C}\right]=\left[B^{\prime}\right]\left[\delta_{A C}\right]+[M A] \cdot\left[\begin{array}{c}
{[d(x c)]^{-1} \cdot[M A]^{t} \cdot \delta} \\
-[d(\lambda)] \cdot[d(x c)]^{-1} \cdot[M D]^{t} \cdot V
\end{array}\right]} \\
{\left[P_{D C}\right]=[G][V]+[M D]\left[\begin{array}{c}
-[d(x c)]^{-1} \cdot[M A]^{t} \cdot \delta \\
+[d(\lambda)] \cdot[d(x c)]^{-1} \cdot[M D]^{t} \cdot V
\end{array}\right]}
\end{gathered}
$$

Eq. (18) and (19) may be combined to be written in a Matrix form as below:

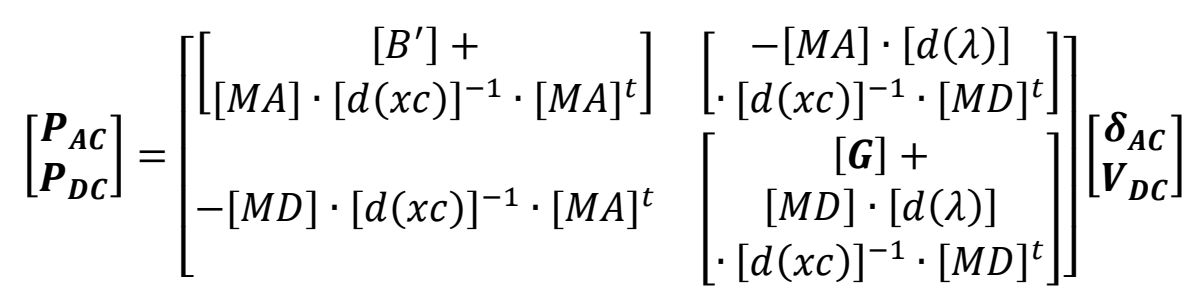

It can be reduced to:

$$
\left[\begin{array}{l}
P_{A C} \\
P_{D C}
\end{array}\right]=\left[\begin{array}{ll}
{[B G 1]} & {[B G 2]} \\
{[B G 3]} & {[B G 4]}
\end{array}\right]\left[\begin{array}{l}
\delta_{A C} \\
V_{D C}
\end{array}\right]
$$

Collating various submatrices, a single matrix is created: $[\boldsymbol{B G}]$ as below:

$$
\left[\begin{array}{l}
\boldsymbol{P}_{A C} \\
\boldsymbol{P}_{D C}
\end{array}\right]=[B G]\left[\begin{array}{l}
\boldsymbol{\delta}_{A C} \\
V_{D C}
\end{array}\right]
$$

The only unknown value in (2.21) or (2.23) is $\lambda$ whose calculation procedure is explained in the next section. 


\subsection{Proposed Three-Step Algorithm}

Equation (2.23) presents the equation of linear AC-DC power-flow analysis method. It means that having the nodal active power injection vector and also the $[\boldsymbol{B G}]$ matrix, the unknown variables of an AC-DC network can be calculated. The $[\boldsymbol{B G}]$ matrix, shown in matrix equation (2.21), includes the reactances and resistances of the AC and DC lines, as well as the parameter $\lambda$. The latter is an unknown parameter and should be calculated to fully establish the $[\boldsymbol{B G}]$ matrix.

In this section, the procedure of the proposed method for linear AC-DC power flow analysis is described. The procedure of the proposed linear AC-DC power-flow analysis method includes three steps, which includes procedure to compute $[\boldsymbol{B} \boldsymbol{G}]$ matrix based on the known parameters of the AC-DC networks and then to solve (2.23).

The first step solves the whole network considering it as a linear AC network, yielding bus phase angles at all busses. The second step computes attributes of the proposed linear model of all AC-DC power converters. Here, the procedure for obtaining the parameter $\lambda$ is introduced.

The third step solves the linear model of the AC-DC system including converters, yielding bus phase angles at AC busses and voltage magnitudes at DC busses. In this third step, the $\mathrm{AC}$ sections of full $\mathrm{AC}-\mathrm{DC}$ power system are represented by real powers and bus phase angles related by respective bus susceptance matrices, the DC sections are represented by real power and bus voltage magnitudes related by respective conductance matrices, and relations between $\mathrm{AC}$ and $\mathrm{DC}$ section busses are related by respective $\lambda$ parameters representing linear models of AC-DC power converters. Aggregating these AC, DC and AC-DC relations, a set of linear power flow equations is presented in a matrix form to represent the entire AC-DC 
network. This matrix is referred in this paper to as [BG] matrix. Finally, having [BG] matrix and the nodal active power injection vector at both AC and DC busses, the unknown variables of the AC-DC linear power flow analysis, which are phase angles of voltage phasors at AC busses and voltage magnitudes at DC busses, can be calculated. The known and unknown variables of the AC-DC power system are listed below.

Table 2.1: Comparison of inputs and output of linear power-flow analysis between AC and DC subgrids

\begin{tabular}{|c|c|c|}
\hline Variables and parameters & AC subgrid & DC subgrid \\
\hline Unknown variable in linear power-flow analysis & Phase angle, $\delta$ & Voltage magnitude, $V$ \\
\hline Network parameter & Reactance, $x$ & Resistance, $r$ \\
\hline Connected load & Active power, $P$ & Active power, $P$ \\
\hline
\end{tabular}

\section{Step \#1- AC only solution}

Without the knowledge of $\lambda$ parameter of linear model of AC-DC power converters, [BG] matrix cannot be formed. To address this, steps \#1 and \#2 are used to compute the $\lambda$ parameter.

For this purpose, the DC sub-grid should be modified in this step \#1 to be treated as a AC network. For this purpose, each resistive element of the DC sub-grid is represented by a reactance element of equal impedance and real power loads at DC busses are retained as real power loads at AC busses.

Now, the original AC-DC network becomes a pure AC network, which is referred to as modified network in this study and it bus-wise susceptance matrix is denoted as $\left[\boldsymbol{B}_{\text {mod }}^{\prime}\right]$. The 
linear AC power flow can be run by using the same nodal active power injection vector as presented in (22). Then, considering the $c^{\text {th }}$ AC-DC power converter connecting the $i^{\text {th }}$ bus of the AC sub-grid and $\mathrm{k}^{\text {th }}$ bus of the DC sub-grid, the value of $\delta_{x}$ is equal to the AC linear power flow result corresponding to the bus which accounts for the DC side of the converter.

\section{Step \#2- Determining $\lambda$ parameter for AC-DC power converters}

Considering (2.12) and assuming that the value of $V_{k}$ is equal to 1 p.u., the value of $\lambda_{c}$ can be calculated having the value of $\delta_{x}$ obtained in the previous step. It should be noted that the value of $\lambda$ must be determined for each AC-DC power converter of the AC-DC network.

\section{Step \#3 - Linear AC-DC power flow}

Determining the value of $\lambda$ for all AC-DC power converters, [BG] matrix can be completely formed. This matrix can represent the entire AC-DC network. Using the nodal active power injection vector (which is the same as used in the first step), (2.23) can be solved for the unknown variables. These variables are phase angles of AC bus voltage phasors and magnitude of voltage at all DC busses. The compact form of the equation for solving (2.47) is written below:

$$
\left[\begin{array}{c}
\boldsymbol{\delta}_{A C} \\
V_{D C}
\end{array}\right]=[B G]^{-1}\left[\begin{array}{l}
P_{A C} \\
P_{D C}
\end{array}\right]
$$

The flowchart of the proposed three-step linear AC-DC power flow method is shown in Figure 2.4. Furthermore, a numerical example of a 4-bus test system is presented in next chapter to demonstrate the procedure of the proposed method. 


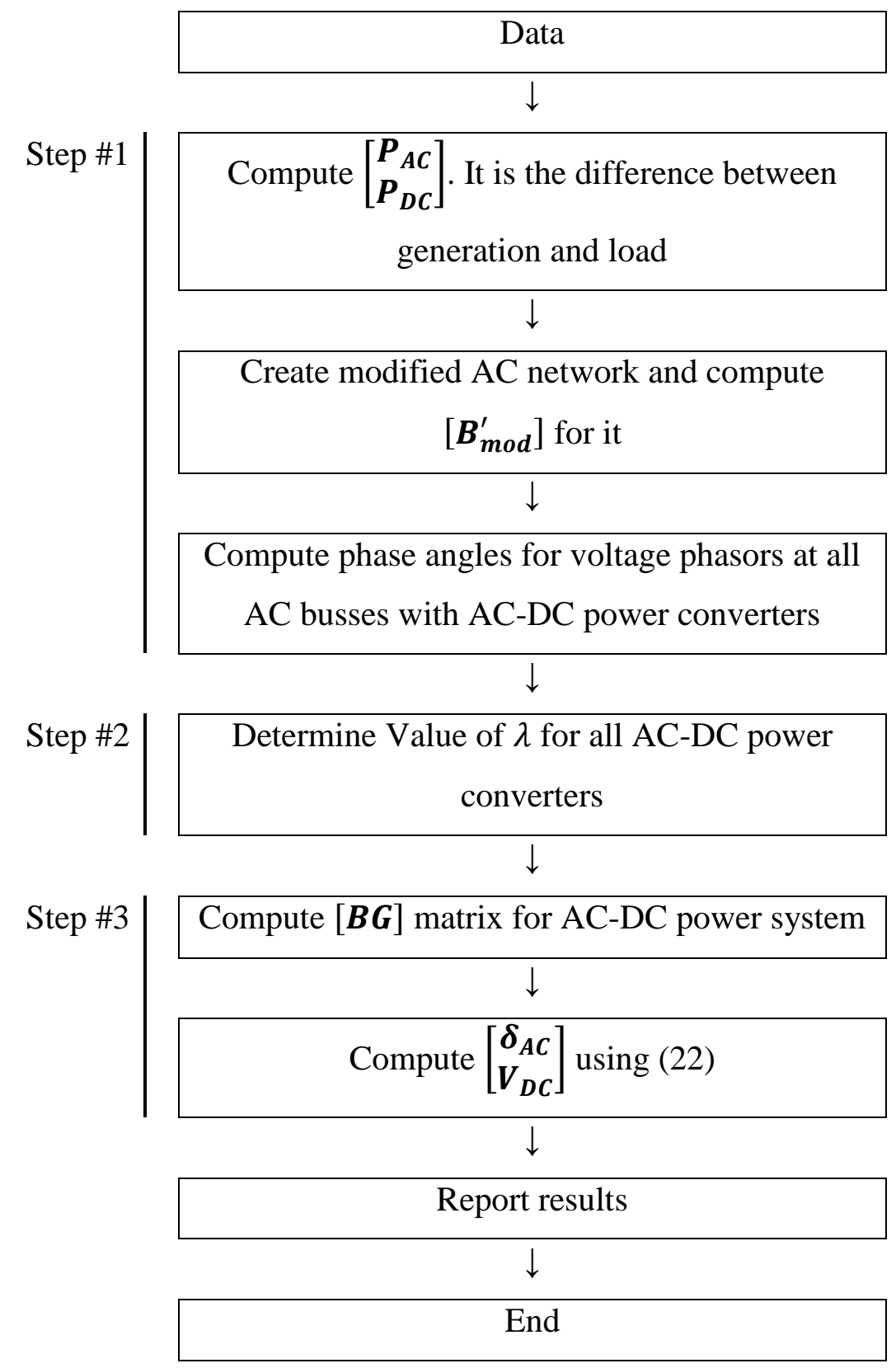

Figure 2.4: Flowchart of the proposed method for linear AC-DC power-flow analysis 


\subsection{Chapter Summary}

In this chapter the problem associated with linear AC-DC power-flow analysis and the proposed solution were presented. In the first part, the problem statement was explained. In order to have a clear understanding of the problem, a brief review of linear power-flow analysis methods for AC and DC networks were given. The assumptions and formulations of those methods were also presented. It was mentioned that the entire $\mathrm{AC}$ and DC networks can be represented by B-prime and G-prime matrices, respectively in the linear power-flow analysis. Then, the existence of AC-DC power converter in an AC-DC network was considered. A new matrix, $[\boldsymbol{B G}]$ matrix, was introduced which represents the entire AC-DC network and relates the nodal power injection vector to the unknown variables of AC-DC networks.

In the second part, the proposed method for linear AC-DC power-flow analysis and its basics and assumptions were presented. First of all, the linear model of AC-DC power converter was suggested based on which the linear equations of active power flowing through the converter were given. The model was based on the relationship between the phase angle of AC side voltage and magnitude of DC side voltage of the ideal AC-DC converter. The relationship parameter was called $\lambda$. Then, the linear model of AC-DC power converter was incorporated in a typical AC-DC network and finally, the equation of linear AC-DC powerflow analysis was yielded. In this equation the elements of $[\boldsymbol{B} \boldsymbol{G}]$ matrix were formulated. The only unknown parameter in $[\boldsymbol{B G}]$ matrix is $\lambda$ which should be determined to enable us to calculate the $[\boldsymbol{B G}]$ matrix.

The last part of this chapter was devoted to the procedure of the proposed linear AC-DC power-flow analysis method. The procedure consists of three steps. In The first step solves the 
whole network considering it as a linear AC network, yielding bus phase angles at all busses. The second step computes attributes of the proposed linear model of all AC-DC power converters. Here, the procedure for obtaining the parameter $\lambda$ is introduced.

The third step solves the linear model of the AC-DC system including converters, yielding bus phase angles at AC busses and voltage magnitudes at DC busses. In this third step, the AC sections of full AC-DC power system are represented by real powers and bus phase angles related by respective bus susceptance matrices, the DC sections are represented by real power and bus voltage magnitudes related by respective conductance matrices, and relations between AC and DC section busses are related by respective $\lambda$ parameters representing linear models of AC-DC power converters. Aggregating these AC, DC and AC-DC relations, a set of linear power flow equations is presented in a matrix form to represent the entire AC-DC network. This matrix is referred in this paper to as [BG] matrix. Finally, having [BG] matrix and the nodal active power injection vector at both AC and DC busses, the unknown variables of the AC-DC linear power flow analysis, which are phase angles of voltage phasors at AC busses and voltage magnitudes at DC busses, can be calculated. 


\section{ChAPTER 3.}

\section{SimUlation RESULTS}

\subsection{Introduction}

In this chapter, the simulation results of the proposed method are presented and its performance is investigated. As the first case study, a numerical example of a 4-bus test system is given to clarify the procedure of the proposed method. It helps to completely understand the step which should be taken for running the proposed linear AC-DC power-flow analysis method. All three steps of the proposed method are demonstrated with detailed calculations and explanations. The 4-bus test system is implemented in MATLAB/Simulink in a balanced condition. The AC-DC power converter is controlled by voltage oriented controller. The results of the proposed method are compared with both MATLAB/Simulink and an accurate non- 
linear AC-DC power flow method. The latter is programmed in MATLAB software where the admittance matrix is formed based on the $\pi$-model of the AC-DC converters and then, NewtonRaphson method is used to solve the non-linear equations of the AC-DC power-flow analysis.

In the next parts, the same procedure of 4-bus test system is used to simulate some larger test systems including more AC-DC power converters and DC lines. Moreover, three defined indices are employed to represent the performance of the proposed method. MATLAB software is utilized to carry out the simulation for all test systems and for both linear proposed method and the accurate non-linear AC-DC power flow method. The results of the proposed linear method are compared with the results of the accurate non-linear AC-DC power flow method [11].

The proposed linear AC-DC power flow method is applied to seven modified test systems including IEEE test systems. The original test systems include IEEE test systems 9, 14, 30, 57, 118, and 300 buses as well as New England 39-bus test system. These standard test systems do not have DC subgrids and AC-DC power converters. Therefore, the original standard test systems are not AC-DC networks. Hence, they are modified in this thesis such that they can be utilized as AC-DC networks. One or more DC subgrids are added to the above-named AC test systems to make them as AC-DC test systems. The number of AC-DC power converters and the DC lines which are added to the original test systems are tabulated in Table 3.1. The parameters of the added components are presented in the related part of each test system. In those test systems with more than one DC subgrid, the $T$ values of the AC-DC power converters are chosen such that a wide range of voltage magnitudes at DC side of the 
converters are obtained. This ensures that the accuracy of the proposed method is checked out in worst cases.

Table 3.1: Summary of the modified test systems

\begin{tabular}{|c|c|c|}
\hline $\begin{array}{c}\text { No. of Buses } \\
\text { in Original network }\end{array}$ & $\begin{array}{c}\text { No. of Added } \\
\text { Converter }\end{array}$ & $\begin{array}{c}\text { No. of Added } \\
\text { DC Line }\end{array}$ \\
\hline 9 & 1 & 5 \\
\hline 14 & 1 & 7 \\
\hline 30 & 2 & 7 \\
\hline 39 & 2 & 12 \\
\hline 57 & 3 & 14 \\
\hline 118 & 4 & 25 \\
\hline 300 & 6 & 30 \\
\hline
\end{tabular}

\subsection{Numerical Example of 4-Bus Test System}

The test system is shown in Figure 3.1. The impedance of transmission line $Z_{12}$ connecting Bus 1 to Bus 2 is $0.008+0.06 \mathrm{i}$ p.u. The DC line resistance $Z_{34}$ between Bus 3 and Bus 4 is 0.012687 in p.u. Moreover, two loads $D_{2}$ and $D_{4}$ are connected at Bus 2 and Bus 4 which are $0.6+0.03 i$ and 0.1844 in p.u., respectively. The magnitude and phase angle of the voltage at Bus 1 as the slack bus are fixed at 1 p.u. and 0 degrees. The AC-DC power converter impedance is $0.0052+0.261$ i p.u. and the voltage transfer ratio $T$ of the ideal AC-DC power converter is equal to $0.9973\left\llcorner-5.6^{\circ}\right.$. 

Bus 1
Bus 2
Bus 3
Bus 4

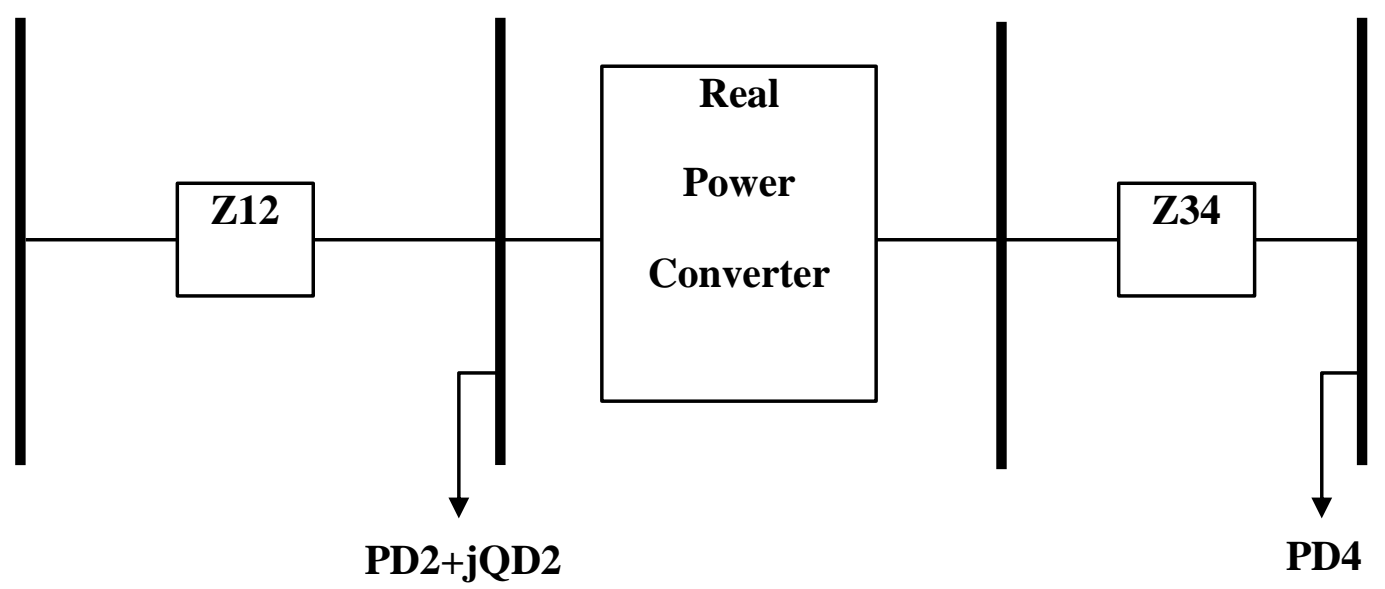

Figure 3.1: Schematic diagram of 4-bus test system

The following three steps should be taken to run the proposed linear AC-DC power flow.

Step \#1: The AC-DC power converter is presented by its equivalent reactance in this step (i.e., 0.261 p.u.). Moreover, the $\mathrm{AC}$ line should be considered just by its reactance due to the assumption of high $X / R$ ratio in $\mathrm{AC}$ linear power-flow analysis. The aim of this step is to establish the modified network and run the AC linear power-flow analysis method for the entire modified network. In the modified network, DC line resistance is replaced by a reactance with the same value. It should be done just to provide the conditions for AC linear power-flow analysis. It means that the modified network resembles a pure AC network for which AC linear power-flow analysis method can be utilized. It should be noticed again although the appearance of the DC line component (i.e., resistance) changes, its value is kept unchanged as the connected loads' values do not change. This guarantees that the results of AC linear powerflow analysis method for AC subgrid will not be affected by this modification. 
Then, the modified B-prime matrix should be formed for the modified network. The elements of the B-prime matrix are calculated below:

$$
\begin{gathered}
b_{11}=\frac{1}{x_{12}}=\frac{1}{0.06}=16.67 \\
b_{12}=b_{21}=\frac{-1}{x_{12}}=\frac{-1}{0.06}=-16.67 \\
b_{22}=\frac{1}{x_{12}}+\frac{1}{x_{C}}=\frac{1}{0.06}+\frac{1}{0.261}=20.497 \\
b_{2 x}=b_{x 2}=\frac{-1}{x_{C}}=\frac{-1}{0.261}=-3.83 \\
b_{x x} \frac{1}{x_{34}}+\frac{1}{x_{C}}=\frac{1}{0.012687}+\frac{1}{0.261}=82.65 \\
b_{x 4}=b_{4 x}=\frac{-1}{x_{34}}=\frac{-1}{0.012687}=-78.82 \\
b_{44}=\frac{1}{x_{34}}=\frac{1}{0.012687}=78.82
\end{gathered}
$$

Now, the B-prime matrix of the modified network of 4-bus test system can be established based on the above calculations. This matrix is shown below for the 4-bus test system.

$$
\left[B_{\text {mod }}^{\prime}\right]=\left[\begin{array}{cccc}
16.67 & -16.67 & 0 & 0 \\
-16.67 & 20.497 & -3.83 & 0 \\
0 & -3.83 & 82.65 & -78.82 \\
0 & 0 & -78.82 & 78.82
\end{array}\right]
$$

As another input for the AC linear power-flow analysis method, the nodal power injection vector should be calculated in this step. This vector is defined as the active power generation minus the active power demand at each bus of the AC-DC network, except the slack bus \#1 as presented in (3.9). 


$$
\begin{gathered}
{\left[\begin{array}{l}
P_{2} \\
P_{3} \\
P_{4}
\end{array}\right]=\left[\begin{array}{l}
P_{2}^{\text {Gen } .}-P_{2}^{\text {Den } .} \\
P_{3}^{\text {Gen }}-P_{3}^{\text {Den. }} \\
P_{4}^{\text {Gen }}-P_{4}^{\text {Den. }} .
\end{array}\right] \text { p.u. }} \\
{\left[\begin{array}{l}
P_{2} \\
P_{3} \\
P_{4}
\end{array}\right]=\left[\begin{array}{c}
-0.6 \\
0 \\
-0.1844
\end{array}\right] \text { p.u. }}
\end{gathered}
$$

Having the modified B-prime matrix in (3.8) and active power injection matrix in (3.10), AC linear power flow can be solved for the phase angles as the unknown variables based on (3.11) which are numbered in (3.12).

$$
\begin{gathered}
{\left[\begin{array}{l}
P_{2} \\
P_{3} \\
P_{4}
\end{array}\right]=\left[B_{\text {Mod }}^{\prime}\right] \times\left[\begin{array}{l}
\delta_{2} \\
\delta_{3} \\
\delta_{4}
\end{array}\right]} \\
{\left[\begin{array}{c}
-0.6 \\
0 \\
-0.1844
\end{array}\right]=\left[\begin{array}{ccc}
20.497 & -3.83 & 0 \\
-3.83 & 82.65 & -78.82 \\
0 & -78.82 & 78.82
\end{array}\right] \times\left[\begin{array}{l}
\delta_{2} \\
\delta_{3} \\
\delta_{4}
\end{array}\right]}
\end{gathered}
$$

By considering the Bus 1 as the slack bus, the unknown variables in (3.12) can be obtained by:

$$
\left[\begin{array}{l}
\delta_{2} \\
\delta_{3} \\
\delta_{4}
\end{array}\right]=\left[\begin{array}{ccc}
20.497 & -3.83 & 0 \\
-3.83 & 82.65 & -78.82 \\
0 & -78.82 & 78.82
\end{array}\right]^{-1} \times\left[\begin{array}{c}
-0.6 \\
0 \\
-0.1844
\end{array}\right]
$$

The calculated result is given below:

$$
\left[\begin{array}{l}
\delta_{2} \\
\delta_{3} \\
\delta_{4}
\end{array}\right]=\left[\begin{array}{l}
-0.0471 \\
-0.0952 \\
-0.0975
\end{array}\right] \text { radian }
$$

At the end of this step, the value of $\delta_{3}$ is determined which is equal to -0.0952 .

Step \#2: In this step, the value of $\lambda$ for the AC-DC power converter is determined. The $\lambda$ value is calculated based on (2.12) while the value of $V_{k=3}$ is assumed to be equal to 1 p.u. in this numerical example. Therefore, the value of $\lambda$ is equal to -0.0952 . This value is required for 
the next step to form the $[\boldsymbol{B} \boldsymbol{G}]$ matrix.

Step \#3: By considering (2.21) and the determined value of $\lambda$ in the previous step, $[\boldsymbol{B} \boldsymbol{G}]$ matrix can be established. The schematic diagram of the test system in this step is depicted in Figure 3.2 where the AC-DC power converter is represented by its equivalent reactance $x_{C}$. The ideal AC-DC converter is modelled and implemented in $[\boldsymbol{B G}]$ matrix and hence it is not shown in this diagram. Moreover, in spite of the modified 4-bus test, the DC line in the third step is represented by its resistance in Figure 3.2.

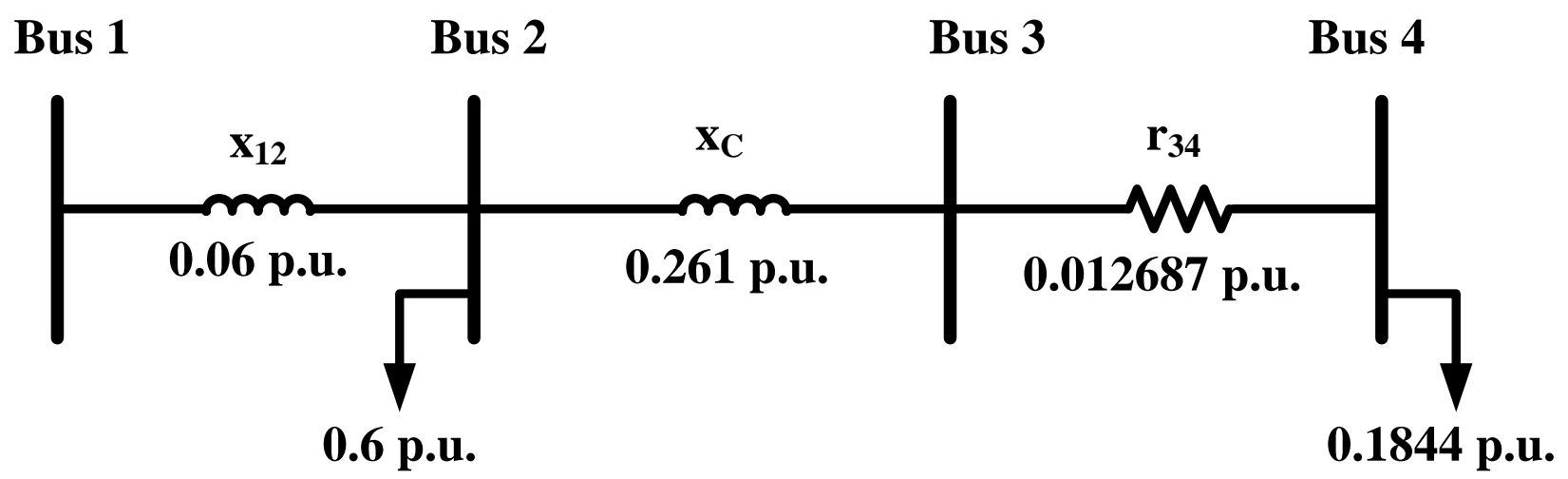

Figure 3.2: Equivalent circuit of the 4-bus test system in the third step of the proposed method

Considering the schematic diagram of the 4-bus test system in the third step of the proposed method as shown in Figure 3.2 and the $[\boldsymbol{B G}]$ matrix equation presented in $(2.21)$, the elements of the $[\boldsymbol{B} \boldsymbol{G}]$ matrix are calculated below:

$$
\begin{gathered}
b g_{11}=\frac{1}{x_{12}}=\frac{1}{0.06}=16.67 \\
b g_{12}=b g_{21}=\frac{-1}{x_{12}}=\frac{-1}{0.06}=-16.67
\end{gathered}
$$




$$
\begin{gathered}
b g_{22}=\frac{1}{x_{12}}+\frac{1}{x_{C}}=\frac{1}{0.06}+\frac{1}{0.261}=20.497 \\
b g_{23}=\frac{-\lambda}{x_{C}}=\frac{-0.0952}{0.261}=0.3646 \\
b g_{32}=\frac{-1}{x_{C}}=\frac{-1}{0.261}=-3.83 \\
b g_{33}=\frac{1}{r_{34}}+\frac{\lambda}{x_{C}}=\frac{1}{0.012687}+\frac{-0.0952}{0.261}=78.46 \\
b g_{34}=b g_{43}=\frac{-1}{r_{34}}=\frac{-1}{0.012687}=-78.82 \\
b g_{44}=\frac{1}{r_{34}}=\frac{1}{0.012687}=78.82
\end{gathered}
$$

Based on the above calculations, the $[\boldsymbol{B G}]$ matrix is written in (3.23).

$$
[B G]=\left[\begin{array}{cccc}
16.67 & -16.67 & 0 & 0 \\
-16.67 & 20.497 & 0.3646 & 0 \\
0 & -3.83 & 78.46 & -78.82 \\
0 & 0 & -78.82 & 78.82
\end{array}\right]
$$

As presented in (3.23), only the two boxed elements of $B G^{\prime}$ matrix are changed in comparison with the modified $\left[B^{\prime}\right]$ matrix. The rows of these modified elements are the bus numbers corresponding to AC and DC sides of the AC-DC power converter. The column of those boxed elements is the bus number corresponding to the DC side of the AC-DC power converter. These are the elements affected by the parameter $\lambda$. The error of unknown AC variables is caused by the nature of $\mathrm{AC}$ subgrid like $X / R$ ratio and voltage variations rather than the proposed linear AC-DC power-flow analysis method and the assumed parameter $\lambda$.

Using the same nodal power injection vector in the first step as presented in (3.10), the following equations should be solved for the voltage of DC buses and the voltage angles of AC ones. 


$$
\begin{gathered}
{\left[\begin{array}{l}
P_{2} \\
P_{3} \\
P_{4}
\end{array}\right]=\left[B G^{\prime}\right] \times\left[\begin{array}{l}
\delta_{2} \\
V_{3} \\
V_{4}
\end{array}\right]} \\
{\left[\begin{array}{c}
-0.6 \\
0 \\
-0.1844
\end{array}\right]=\left[\begin{array}{ccc}
20.497 & 0.3646 & 0 \\
-3.83 & 78.46 & -78.82 \\
0 & -78.82 & 78.82
\end{array}\right] \times\left[\begin{array}{l}
\delta_{2} \\
V_{3} \\
V_{4}
\end{array}\right]}
\end{gathered}
$$

By considering the Bus 1 as the slack bus, the unknown variables in (3.25) can be obtained by:

$$
\left[\begin{array}{l}
\delta_{2} \\
V_{3} \\
V_{4}
\end{array}\right]=\left[\begin{array}{ccc}
20.497 & 0.3646 & 0 \\
-3.83 & 78.46 & -78.82 \\
0 & -78.82 & 78.82
\end{array}\right]^{-1} \times\left[\begin{array}{c}
-0.6 \\
0 \\
-0.1844
\end{array}\right]
$$

The results of these variables in the third step are as presented in (3.27).

$$
\left[\begin{array}{l}
\delta_{2} \\
V_{3} \\
V_{4}
\end{array}\right]=\left[\begin{array}{c}
-0.0471 \\
0.9999 \\
0.9976
\end{array}\right]
$$

The first element in (3.27) is the phase angle in radian but the others are DC voltage in p.u. By comparing the AC variable $\delta_{2}$ in (3.14) and (3.27), it can be observed that they have the same value (i.e, -0.0471). This confirms the claim that the proposed linear AC-DC power-flow analysis method does not influence the AC subgrid variables. In contrast, the second and the third elements in (3.14) have changed to new values in (3.27). It means that incorporation of the obtained $\lambda$ value (in the second step) in the $[\boldsymbol{B} \boldsymbol{G}]$ matrix (in the third step) has just modified the $\mathrm{DC}$ variables but not $\mathrm{AC}$ variable of the first step. In other words, the errors of $\mathrm{AC}$ variables are almost independent of the proposed linear method and caused by the nature of the AC subgrid and will appeared in linear AC power flow too.

The results of the proposed linear method are compared with time-domain simulation in MATLAB/Simulink environment and the accurate conventional non-linear AC-DC power flow method [11]. 
The results of 4-bus test system are tabulated in Table 3.2. The linear active power flowing through the AC-DC power converter $\left(P_{C A l}\right)$ is calculated based on (2.15) and as follows:

$$
\begin{gathered}
P_{C A 1}=\frac{1}{x c_{c}} \delta_{2}-\frac{\lambda}{x c_{c}} V_{3} \\
P_{C A 1}=\frac{1}{0.261} \times(-0.0471)-\frac{-0.0952}{0.261} \times 0.9999=0.1842
\end{gathered}
$$

The values of flowing active power in AC line $P_{12}$ and DC line $P_{34}$ are computed as follows:

$$
\begin{gathered}
P_{12}=\frac{\delta_{1}-\delta_{2}}{x_{12}}=\frac{0+0.0471}{0.06}=0.785 \\
P_{34}=\frac{V_{3}-V_{4}}{r_{34}}=\frac{0.9999-0.9976}{0.012687}=0.1813
\end{gathered}
$$

As can be seen in Table 3.2, the proposed nonlinear method provides a reasonably accurate model, similar to the linear AC model of power system (called the DC power flow). It is obvious that this linear model can be easily integrated into complex planning and operational optimization formulations for AC-DC power systems providing benefits in computation speed.

Table 3.2: The results of numerical example of 4-bus test system

\begin{tabular}{|l|c|c|c|c|}
\hline Variable & Units & $\begin{array}{c}\text { MATLAB/ } \\
\text { Simulink }\end{array}$ & $\begin{array}{c}\text { Accurate Nonlinear } \\
\text { Power Flow }\end{array}$ & $\begin{array}{c}\text { Proposed Linear } \\
\text { Power Flow }\end{array}$ \\
\hline$\delta_{2}$ & Radians & -0.04747 & -0.04758 & -0.0471 \\
\hline$V_{3}$ & Per unit & 1.001 & 0.9943 & 0.9999 \\
\hline$V_{4}$ & Per unit & 0.9982 & 0.992 & 0.9976 \\
\hline$P_{12}$ & Per unit & 0.793 & 0.79 & 0.785 \\
\hline$P C A_{1}$ & Per unit & 0.189 & 0.1833 & 0.1842 \\
\hline$P_{34}$ & Per unit & 0.1844 & 0.1848 & 0.1813 \\
\hline
\end{tabular}




\subsection{Modified IEEE 9-Bus Test System}

In the previous section, the procedure of the proposed linear AC-DC power-flow analysis method was applied to a 4-bus test system. The detail explanations and calculations were presented to make the procedure more clearly comprehensible. Now, the same procedure is applied to a larger network. In this section, IEEE 9-bus test system is used for investigating the proposed linear AC-DC Power-flow analysis method. However, the original aforementioned test system is a pure $\mathrm{AC}$ network and it requires modification to be prepared for linear AC-DC power-flow analysis. In this regard, one AC-DC power converter and 5 DC lines are added to the original IEEE 9-bus test system. The AC-DC power converter is connected to the bus number 8 of the original test system. The data for IEEE 9-bus test system can be found in [34]. In addition, the bus data and the DC lines resistances added to the original test system are shown in Table A.1. The AC-DC power converter impedance is $0.008+0.05 \mathrm{i}$ p.u. connected between buses 8 in AC subgrid and 10 in DC subgrid. Since the results of the proposed AC-DC power-flow analysis method are compared with the results of the accurate nonlinear AC-DC power flow method, the parameter $T$ should be known as an input variable [11]. This value is equal to $1.07 \Varangle-0.6$ for the used AC-DC power converter in this test system. This value is chosen such that the voltage value of 1.003 p.u. is produced at DC side of the AC-DC power converter.

Figure 3.3 shows the comparison of phase angle error of $\mathrm{AC}$ buses between linear AC and AC-DC power flow methods for the modified IEEE 9-bus test system. The results of the linear AC power-flow analysis method for the original IEEE 9-bus test system are shown in this figure. As the AC subgrid in the modified IEEE-9-bus test system, the nature of the original 
IEEE 9-bus test system is almost kept unchanged. Then, the proposed linear AC-DC powerflow analysis method is applied to the modified test system. The phase angle of AC bus voltages are shown in Figure 3.3. This figure indicates that the $\mathrm{AC}$ variables are not considerably affected by the proposed linear AC-DC power-flow analysis method.

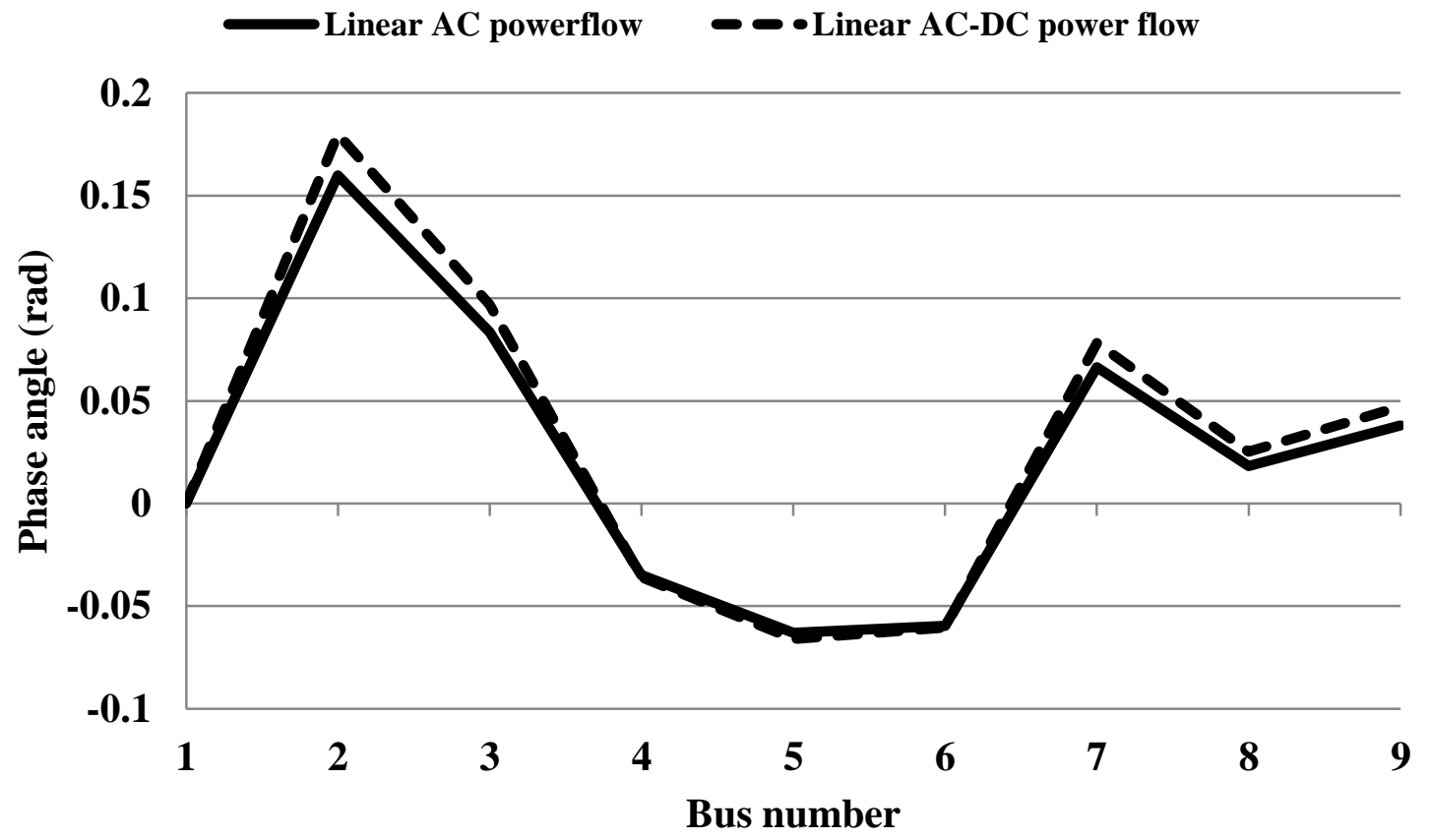

Figure 3.3: Comparison of phase angle error of AC buses between linear AC and AC-DC power flow methods for the modified IEEE 9-bus test system

In order to support the above-mentioned sentence, the comparison of power flow error of AC lines between linear AC and AC-DC power flow methods for the modified IEEE 9-bus test system is depicted in Figure 3.4. In this figure, the power flow error corresponding to linear AC power flow is calculated based on the linear AC power-flow analysis method and NewtonRaphson method. Furthermore, the power flow error corresponding to linear AC-DC power flow is calculated based on the proposed linear AC-DC power-flow analysis method and the accurate non-linear AC-DC power flow [11]. It is obvious in this figure that the linear AC 
power flow in the original IEEE 9-bus test system and the proposed linear AC-DC power-flow analysis method in the modified test system have almost the same results. Therefore, the error of power flow in AC subgrid does not come from the proposed method in this thesis and is caused by the AC subgrid topology and parameters.

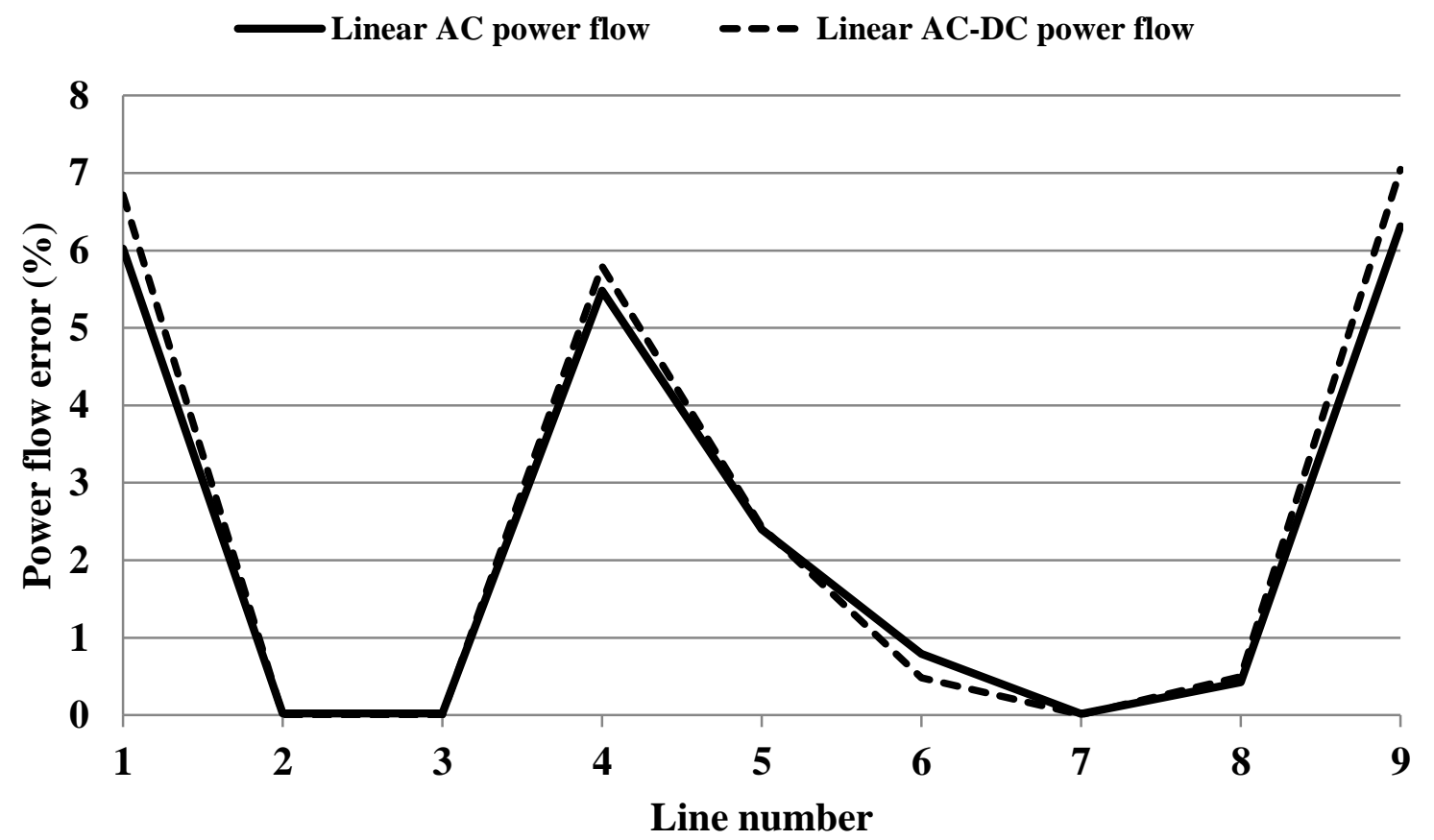

Figure 3.4: Comparison of power flow error of AC lines between linear AC and AC-DC power flow methods for the modified IEEE 9-bus test system

\subsection{Modified IEEE 14-Bus Test System}

As another test system, IEEE 14-bus network is used in this section. Like the previous test system, IEEE 14-bus network is a pure AC network and should be modified for being utilized in AC-DC power flow studies. IEEE 14-bus test system data is available at [35]. Table A.2 presents the data of the added buses and DC lines to the original IEEE 14-bus test system. One AC-DC power converter connects the AC subgrid (i.e., original IEEE 14-bus test system) to the 
DC subgrid (i.e., the added DC buses and lines). The AC-DC power converter impedance is $0.03181+0.0845 \mathrm{i}$ p.u. connected between buses 8 in AC subgrid and 10 in DC subgrid. In this example, the $X / R$ ration of the AC-DC power converter impedance is close to 2.66 which is less than 4. The AC-DC power converter transfer ratio $T$ is equal to 1.02 $\measuredangle-12.773$ for the used ACDC power converter in this test system. This value is chosen such that the voltage value of 1.0107 p.u. is produced at DC side of the AC-DC power converter.

The simulation results related to the modified IEEE 14-bus test system are tabulated in Table 3.3. The AC and DC variables are phase angle and magnitude of bus voltages, respectively. The results of the proposed linear AC-DC power-flow analysis method are compared with the results obtained from accurate nonlinear AC-DC power flow method. The bus numbers from 1 to 14 correspond to AC subgrid and from 15 to 22 correspond to DC subgrid. The last column of Table 3.3 expresses the percent error proposed linear AC-DC power-flow analysis method with respect to the accurate nonlinear AC-DC power flow method. As can be observed in this table, the error of DC variables is limited almost to $1 \%$ while the $\mathrm{AC}$ variables have greater error value. It should be noted again that the error of $\mathrm{AC}$ variables is almost caused by the $\mathrm{AC}$ subgrid itself and the proposed linear AC-DC power-flow analysis method does not play a noticeable role. However, the error of DC variables is totally caused by the proposed method but is negligible. The range of the error for DC variables stays low while the variables vary from 1 p.u. to 0.95 p.u. Therefore, the performance of the proposed linear AC-DC power-flow analysis method is not influenced by the voltage of the DC buses. 
Table 3.3: Comparison of AC and DC variables results between the proposed linear AC-DC power flow method and the accurate non-linear power flow method

\begin{tabular}{|c|c|c|c|c|c|}
\hline $\begin{array}{c}\text { Bus } \\
\text { No. }\end{array}$ & Bus Type, Variable & Unit & $\begin{array}{c}\text { Accurate } \\
\text { Nonlinear } \\
\text { Power }(\mathbf{M W})\end{array}$ & $\begin{array}{c}\text { Proposed Linear } \\
\text { AC-DC } \\
\text { Power flow }\end{array}$ & Error \% \\
\hline 1 & AC, Phase Angle & radian & 0 & 0 & 0 \\
\hline 2 & AC, Phase Angle & radian & -0.08562 & -0.08611 & -0.57014 \\
\hline 3 & AC, Phase Angle & radian & -0.21992 & -0.22378 & -1.75419 \\
\hline 4 & AC, Phase Angle & radian & -0.17632 & -0.18161 & -3.00097 \\
\hline 5 & AC, Phase Angle & radian & -0.15032 & -0.15585 & -3.68484 \\
\hline 6 & AC, Phase Angle & radian & -0.24504 & -0.25268 & -3.11874 \\
\hline 7 & AC, Phase Angle & radian & -0.22637 & -0.23699 & -4.69125 \\
\hline 8 & AC, Phase Angle & radian & -0.22637 & -0.23699 & -4.69125 \\
\hline 9 & AC, Phase Angle & radian & -0.25272 & -0.26677 & -5.56196 \\
\hline 10 & AC, Phase Angle & radian & -0.25629 & -0.27176 & -6.03428 \\
\hline 11 & AC, Phase Angle & radian & -0.25277 & -0.26581 & -5.15855 \\
\hline 12 & AC, Phase Angle & radian & -0.25948 & -0.27132 & -4.56301 \\
\hline 13 & AC, Phase Angle & radian & -0.26033 & -0.27369 & -5.13182 \\
\hline 14 & AC, Phase Angle & radian & -0.27218 & -0.28699 & -5.44277 \\
\hline 15 & DC, Voltage Magnitude & Per-unit & 1.010675 & 1 & 1.056201 \\
\hline 16 & DC, Voltage Magnitude & Per-unit & 1.001072 & 0.990597 & 1.046364 \\
\hline 17 & DC, Voltage Magnitude & Per-unit & 0.989873 & 0.979658 & 1.031932 \\
\hline 18 & DC, Voltage Magnitude & Per-unit & 0.984848 & 0.974763 & 1.024015 \\
\hline 19 & DC, Voltage Magnitude & Per-unit & 0.980281 & 0.970332 & 1.014928 \\
\hline 20 & DC, Voltage Magnitude & Per-unit & 0.97069 & 0.961054 & 0.992762 \\
\hline 21 & DC, Voltage Magnitude & Per-unit & 0.965911 & 0.956438 & 0.980648 \\
\hline 22 & DC, Voltage Magnitude & Per-unit & 0.96535 & 0.955898 & 0.979209 \\
\hline & & & & & \\
\hline
\end{tabular}

In order to analyze the sensitivity of the proposed linear AC-DC power-flow analysis method to different load levels, the modified IEEE 14-bus test system is simulated for three diverse load level: normal load, 1.5 times of the normal load, and 2 times of the normal load. Figure 3.5 illustrates the results of power flow error of the modified test system lines. The error is calculated with respect to the accurate non-linear AC-DC power flow method. It can be seen 
in this figure that the error values of DC lines are less affected by greater load levels than the AC lines. In other words, the proposed method does not have a noticeable sensitivity to the load levels of the AC-DC network. Since the proposed linear AC-DC power-flow analysis method takes care of DC buses and variables, the results of Figure 3.5 confirms that the proposed method has an acceptable performance under different load conditions. It is worth mentioning that the line numbers from 1 to 21 correspond to AC lines while line numbers from 22 to 27 correspond to DC lines and finally line number 28 is assigned to the AC-DC power converter.

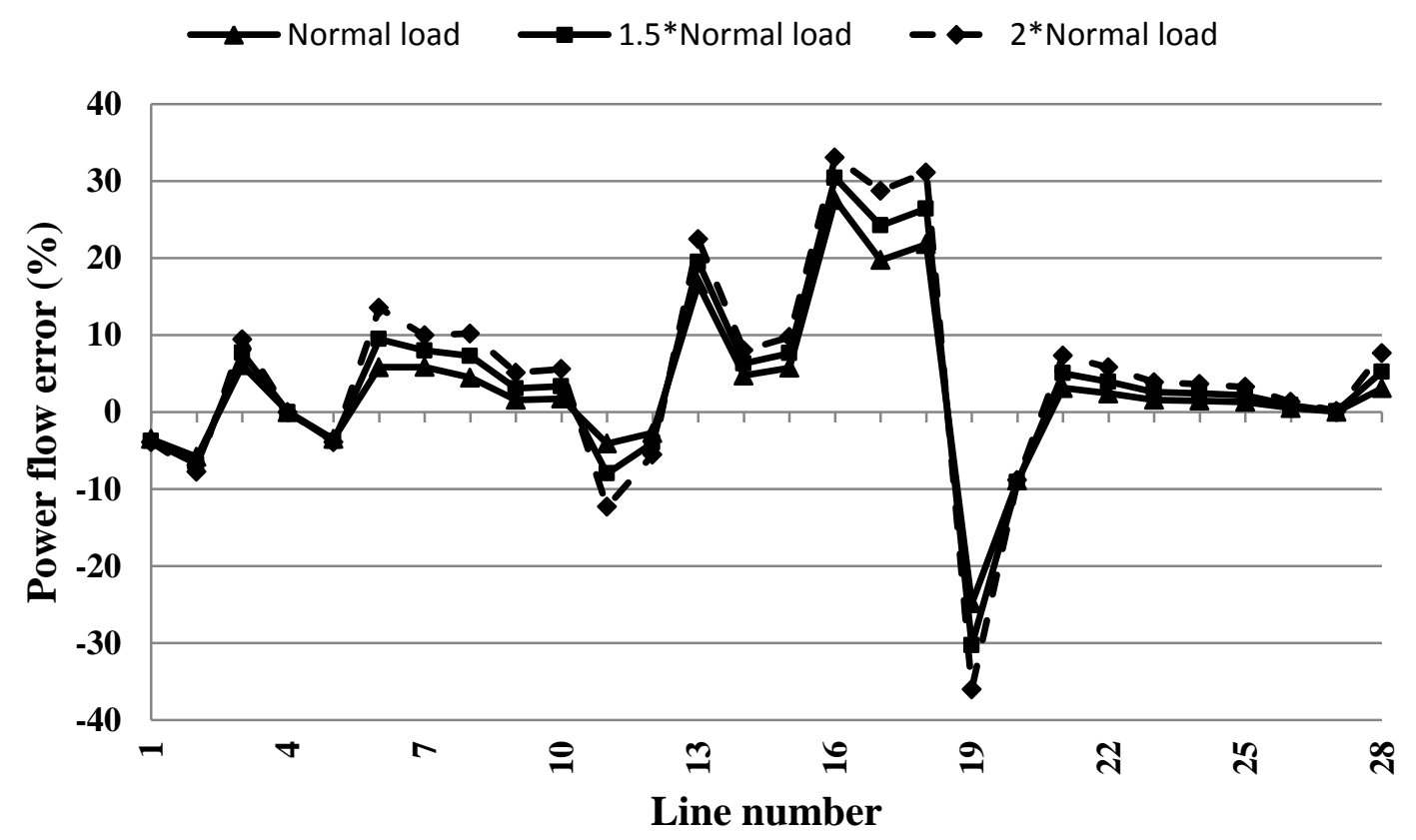

Figure 3.5: Power flow error of the proposed linear AC-DC power-flow analysis method for different load levels of the modified IEEE 14-bus test system section 


\subsection{Modified IEEE 30-Bus Test System}

The original IEEE 30-bus test system data is presented in [36]. This pure AC power system is changed to AC-DC one by adding two AC-DC power converters and 8 DC lines. The data of the added buses and DC lines to the original IEEE 30-bus test system are shown in Table A.3. The AC-DC power converters impedances are $0.267+0.82 \mathrm{i}$ p.u. and $0.12+0.42 \mathrm{i}$ p.u. connected between buses 19 and 30 in AC subgrid as well as 31 and 35 in DC subgrid, respectively. The transfer ratios $\mathrm{T}$ are equal to $1.02 \measuredangle-17$ and $0.98 \measuredangle-18$ for the used AC-DC power converters in this test system. These values are chosen such that the voltage values of 0.9899 p.u. and 1.0135 p.u. are produced at DC sides of the AC-DC power converters which are bus numbers 31 and 35 , respectively.

The results of active power flowing through AC and DC lines of the modified IEEE 30bus test system are arranged in the tabular form of Table 3.4. The results of the proposed linear AC-DC power-flow analysis method are compared in this table with the accurate non-linear AC-DC power flow. The line numbers from 1 to 41 correspond to AC lines while the line numbers from 42 to 48 are assigned to DC lines. In addition, the line numbers 49 and 50 correspond to two AC-DC power converters in the modified IEEE 30-bus test system. The average of absolute values of error in Table 3.4 is equal to $7.05 \%$. It can be observed in Table 3.4 that the absolute values of error of the DC lines and AC-DC power converters are considerably lower than AC lines. This point confirms the robustness of the proposed linear AC-DC power-flow analysis method which is responsible for DC subgrid and AC-DC power converters variables. 
The highest absolute values of error are $87.92 \%, 31.79 \%$, and $26.03 \%$ respectively correspond to the line numbers 34, 33, and 40 all of them are located in AC subgrid. With paying more attention to the power flowing through the aforementioned lines it can be seen that all of them have small values. Numerically, these values are $-1.35 \mathrm{MW}, 1.74 \mathrm{MW}$, and -0.54 MW obtained by the non-linear AC-DC power flow. These three lines have the highest absolute values of error while their actual power flow values are much lower than the average value of power flow among all AC and DC lines which is equal to $20.4 \mathrm{MW}$. In other words, the highest values of error correspond to the AC lines transferring the lowest amount of active power. On the other hand, the absolute value of the error for the AC line number 8, carrying the highest value of active power, is $7.02 \%$.

The above information is collected and presented in Table 3.5. The last two rows express the highest values of transferred power. In this table, the absolute values of difference for line numbers 8 and 9 are too much greater than the same parameter for line numbers 33, 34, and 40 . However, the percent errors of the latter line numbers with lower power flow values are noticeably greater than the percent error of the grayed out rows with higher power flow values. Hence, it can be derived that the percent error is not a perfect index for comparing and analyzing the obtained results.

Considering the above explanations about the issue accounting for percent error, a new parameter is defined below:

$$
P L_{N L}^{A v e}=\sum_{i \in n l} \frac{\left|P L_{N L-i}\right|}{n l}
$$


$P L_{N L}^{A v e}$ is the average of absolute values of power flowing through all lines of AC-DC test system computed using the accurate nonlinear AC-DC power flow method.

Table 3.4: The results of the modified IEEE 30-bus test system

\begin{tabular}{|c|c|c|c|c|c|c|c|}
\hline $\begin{array}{l}\dot{z} \\
\stackrel{\Xi}{\Xi} \\
\stackrel{\Xi}{\Xi}\end{array}$ & 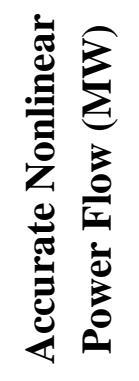 & 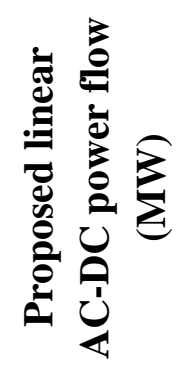 & 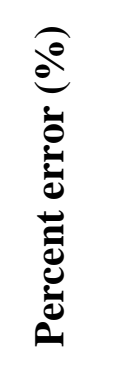 & $\begin{array}{l}\dot{0} \\
\dot{Z} \\
\dot{Z}\end{array}$ & 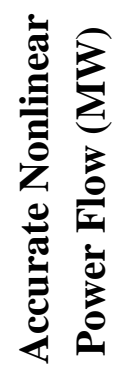 & 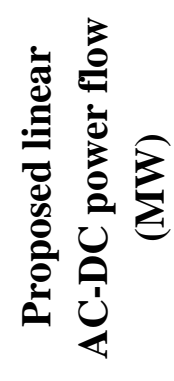 & 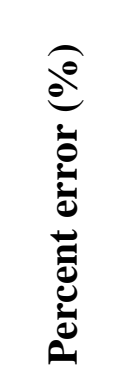 \\
\hline 1 & 27.78 & 27.33 & 1.61 & 26 & 9.19 & 9.11 & 0.87 \\
\hline 2 & 15.89 & 15.90 & 0.09 & 27 & 5.35 & 5.83 & 9.11 \\
\hline 3 & 0.00 & 0.00 & 0.00 & 28 & 15.74 & 15.23 & 3.22 \\
\hline 4 & 27.78 & 27.33 & 1.61 & 29 & 7.59 & 7.25 & 4.39 \\
\hline 5 & 44.13 & 42.44 & 3.83 & 30 & -1.87 & -2.27 & 21.29 \\
\hline 6 & 0.00 & 0.00 & 0.00 & 31 & 4.96 & 4.38 & 11.71 \\
\hline 7 & 18.35 & 19.03 & 3.71 & 32 & 5.67 & 4.99 & 11.97 \\
\hline 8 & 173.19 & 161.03 & 7.02 & 33 & 1.74 & 1.18 & 31.79 \\
\hline 9 & 88.05 & 82.37 & 6.45 & 34 & -1.35 & -2.53 & 87.92 \\
\hline 10 & 43.65 & 42.49 & 2.67 & 35 & 3.54 & 3.50 & 1.24 \\
\hline 11 & 82.52 & 79.97 & 3.09 & 36 & -4.90 & -6.03 & 23.09 \\
\hline 12 & 82.25 & 77.97 & 5.20 & 37 & 6.25 & 6.06 & 2.93 \\
\hline 13 & 60.41 & 58.87 & 2.55 & 38 & 7.18 & 6.94 & 3.41 \\
\hline 14 & 72.58 & 72.42 & 0.22 & 39 & 3.77 & 3.66 & 2.69 \\
\hline 15 & -14.89 & -16.23 & 8.96 & 40 & -0.54 & -0.40 & 26.03 \\
\hline 16 & 38.23 & 39.03 & 2.10 & 41 & 18.95 & 19.43 & 2.53 \\
\hline 17 & 29.56 & 29.60 & 0.13 & 42 & 6.54 & 6.50 & 0.62 \\
\hline 18 & 7.81 & 7.58 & 2.93 & 43 & 4.51 & 4.50 & 0.25 \\
\hline 19 & 17.89 & 16.99 & 5.04 & 44 & 2.00 & 2.00 & 0.12 \\
\hline 20 & 7.23 & 6.67 & 7.76 & 45 & 7.57 & 7.50 & 0.87 \\
\hline 21 & 1.54 & 1.38 & 10.23 & 46 & 5.03 & 5.00 & 0.57 \\
\hline 22 & 3.67 & 3.17 & 13.86 & 47 & 3.01 & 3.00 & 0.35 \\
\hline 23 & 6.05 & 5.79 & 4.36 & 48 & 2.00 & 2.00 & 0.15 \\
\hline 24 & 2.82 & 2.59 & 8.07 & 49 & 8.21 & 8.00 & 2.55 \\
\hline 25 & -6.90 & -6.91 & 0.18 & 50 & 9.10 & 9.00 & 1.05 \\
\hline
\end{tabular}


Table 3.5: The problem of analyzing the results just based on the percent error values

\begin{tabular}{|c|c|c|c|c|}
\hline 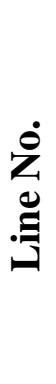 & 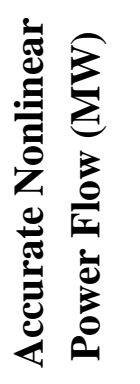 & 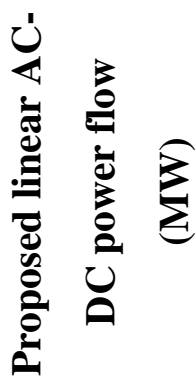 & 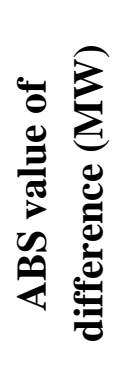 & 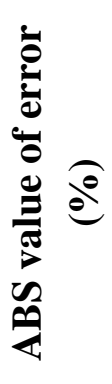 \\
\hline 33 & 1.74 & 1.18 & 0.56 & 31.79 \\
\hline 34 & -1.35 & -2.53 & 1.18 & 87.92 \\
\hline 40 & -0.54 & -0.4 & 0.14 & 26.03 \\
\hline 8 & 173.19 & 161.03 & 12.16 & 7.02 \\
\hline 9 & 88.05 & 82.37 & 5.68 & 6.45 \\
\hline
\end{tabular}

\subsection{Modified New England 39-Bus Test System}

The New England 39-bus test system is another network which is studied in this part. The data of the New England 39-bus test system can be found in [37]. The data of the added buses and DC lines to the original New England 39-bus test system are shown in Table A.4. The original New England 39-bus test system is changed to AC-DC one by adding two AC-DC power converters and 12 DC lines. The AC-DC power converters impedances are $0.0043+0.0474$ i p.u. and $0.0057+0.0625$ i p.u. connected between buses 15 and 27 in AC subgrid as well as 40 and 47 in DC subgrid, respectively. The transfer ratios $T$ are equal to 1.02 Ł-11 and 1.06ð-11 for the used AC-DC power converters in this test system. These values are chosen such that the voltage values of 1.0339 p.u. and 1.0237 p.u. are produced at DC sides of the AC-DC power converters which are bus numbers 40 and 47, respectively. 
Table 3.6 gives the results of active power flowing through the lines of the modified New England 39-bus test system. The results of the proposed linear AC-DC power-flow analysis method are compared in this table with the accurate non-linear AC-DC power flow. Moreover, for AC lines, the results of the proposed linear AC-DC power-flow analysis method are compared with the linear AC power flow method. It should be noted here that the lines with active power flow below the $P L_{N L}^{A v e}$ are ignored. The table confirms that the results of power flow in $\mathrm{AC}$ lines computed by using the proposed linear AC-DC power flow analysis method are very close to the results computed by using the linear AC power flow. The last column of the table shows the percent error between the linear AC-DC power flow and the linear AC power flow methods in AC lines. This value is calculated based on the following equation:

$$
\operatorname{Error}(\%)=100 * \frac{P L_{L i n}-P L_{\text {Lin }}^{A C}}{P L_{L i n}}
$$

where $P L_{\text {Lin }}^{A C}$ is the real power flow in lines computed using the linear AC power flow method.

Table 3.6: The results of the modified IEEE 39-bus test system

\begin{tabular}{|c|c|c|c|c|c|}
\hline $\begin{array}{c}\text { Line } \\
\text { No. }\end{array}$ & $\begin{array}{c}\text { Accurate } \\
\text { Nonlinear } \\
\text { Power Flow } \\
\text { (MW) }\end{array}$ & $\begin{array}{c}\text { Proposed } \\
\text { linear } \\
\text { power flow } \\
(\mathbf{M W})\end{array}$ & $\begin{array}{c}\text { Percent Error - } \\
\text { comparing Proposed } \\
\text { Linear AC-DC power } \\
\text { flow with Accurate } \\
\text { Nonlinear Power Flow } \\
\text { method (\%) }\end{array}$ & $\begin{array}{c}\text { Linear } \\
\text { AC } \\
\text { power } \\
\text { flow } \\
(\mathbf{M W})\end{array}$ & $\begin{array}{c}\text { Percent Error - } \\
\text { comparing Linear } \\
\text { AC power flow } \\
\text { with Accurate } \\
\text { Nonlinear Power } \\
\text { Flow method (\%) }\end{array}$ \\
\hline 1 & -673.737 & -622.032 & 7.674385 & -625.03 & 6.41034 \\
\hline 2 & -650 & -650 & $4.24 \mathrm{E}-06$ & -650 & $9.75 \mathrm{E}-06$ \\
\hline 3 & -605.338 & -632 & -4.4044 & -632 & -4.41928 \\
\hline 4 & -503.143 & -508 & -0.96538 & -508 & -0.96363 \\
\hline 5 & -650 & -650 & $3.73 \mathrm{E}-05$ & -650 & $3.78 \mathrm{E}-05$ \\
\hline 6 & -558.607 & -560 & -0.24941 & -560 & -0.24892 \\
\hline
\end{tabular}




\begin{tabular}{|c|c|c|c|c|c|}
\hline $\begin{array}{c}\text { Line } \\
\text { No. }\end{array}$ & $\begin{array}{c}\text { Accurate } \\
\text { Nonlinear } \\
\text { Power Flow } \\
\text { (MW) }\end{array}$ & $\begin{array}{l}\text { Proposed } \\
\text { linear } \\
\text { power flow } \\
\text { (MW) }\end{array}$ & $\begin{array}{c}\text { Percent Error - } \\
\text { comparing Proposed } \\
\text { Linear AC-DC power } \\
\text { flow with Accurate } \\
\text { Nonlinear Power Flow } \\
\text { method }(\%)\end{array}$ & $\begin{array}{l}\text { Linear } \\
\text { AC } \\
\text { power } \\
\text { flow } \\
\text { (MW) }\end{array}$ & $\begin{array}{l}\text { Percent Error - } \\
\text { comparing Linear } \\
\text { AC power flow } \\
\text { with Accurate } \\
\text { Nonlinear Power } \\
\text { Flow method (\%) }\end{array}$ \\
\hline 7 & -535.184 & -540 & -0.89981 & -540 & -0.90475 \\
\hline 8 & -815.495 & -830 & -1.77872 & -830 & -1.78815 \\
\hline 9 & 319.2542 & 333.7229 & -4.53203 & 333.4301 & -4.37204 \\
\hline 10 & -264.794 & -268.556 & -1.42072 & -268.199 & -0.87781 \\
\hline 11 & -539.364 & -513.175 & 4.855542 & -514.754 & 3.993091 \\
\hline 12 & 339.5923 & 337.0218 & 0.75695 & 337.068 & 0.744902 \\
\hline 13 & 454.4197 & 448.1434 & 1.381167 & 448.4783 & 1.166793 \\
\hline 14 & -320.595 & -339.286 & -5.83026 & -338.202 & -4.82517 \\
\hline 15 & 325.9892 & 341.8916 & -4.8782 & 340.9043 & -3.97219 \\
\hline 16 & 324.0138 & 308.1084 & 4.90886 & 309.0957 & 4.044076 \\
\hline 17 & 319.3511 & 302.1836 & 5.375727 & 303.2679 & 4.424606 \\
\hline 18 & -270.239 & -285.374 & -5.60051 & -284.931 & -5.5251 \\
\hline 19 & -451.608 & -460 & -1.8583 & -460 & -1.85407 \\
\hline 20 & -329.908 & -334.776 & -1.47548 & -334.776 & -1.46612 \\
\hline 21 & -604.71 & -608.776 & -0.67239 & -608.776 & -0.66667 \\
\hline 22 & 353.7564 & 353.7242 & 0.009088 & 353.7242 & 0.005819 \\
\hline 23 & -347.783 & -351.365 & -1.03 & -351.365 & -1.05812 \\
\hline 24 & 272.1243 & 272 & 0.045674 & & \\
\hline 25 & 226.1021 & 226 & 0.045148 & & \\
\hline 26 & 178.0663 & 178 & 0.037211 & & \\
\hline 27 & 135.025 & 135 & 0.01849 & & \\
\hline 28 & 87.0104 & 87 & 0.011952 & & \\
\hline 29 & 52.00589 & 52 & 0.011332 & & \\
\hline 30 & 244.46 & 244 & 0.188166 & & \\
\hline 31 & 205.265 & 205 & 0.129124 & & \\
\hline 32 & 157.2049 & 157 & 0.130346 & & \\
\hline 33 & 121.0964 & 121 & 0.079591 & & \\
\hline 34 & 81.01093 & 81 & 0.013494 & & \\
\hline 35 & 36.00163 & 36 & 0.004534 & & \\
\hline 36 & 323.3414 & 319 & 1.342662 & & \\
\hline 37 & 283.4958 & 279 & 1.585829 & & \\
\hline 38 & -673.737 & -622.032 & 7.674385 & & \\
\hline
\end{tabular}




\subsection{Modified IEE 57-Bus Test System}

In this section, IEEE 57-bus test system is applied for simulation of the proposed linear AC-DC power-flow analysis method. The data of the aforementioned test system is provided in [38]. 14 DC lines and 3 AC-DC power converters are added to the original IEEE 57-bus test system to make it as the modified AC-DC network. The data of the added DC buses and lines are presented in Table A.5. Moreover, the AC-DC power converters data is provided in Table A.6.

Figure 3.6 demonstrates the percent error of power flow of the modified IEEE 57-bus test system lines for the lines which have power flow more than $P L_{N L}^{A v e}$ (except DC lines). It is observable in this figure that maximum percent error between the accurate non-linear AC-DC power flow and the proposed linear AC-DC power-flow analysis method reaches almost $12 \%$ in an $\mathrm{AC}$ line. However, for $\mathrm{DC}$ lines and the $\mathrm{AC}-\mathrm{DC}$ power converters the error value is small.

In order to show the fact that the error in $\mathrm{AC}$ lines are caused by the nature of the $\mathrm{AC}$ subgrid and not by the proposed linear AC-DC power-flow analysis method, the mismatch between linear AC-DC and linear AC power flow values in the modified IEEE 57-bus test system is demonstrated in Figure 3.7. This figure is obtained just for the lines with power flow values more than $P L_{N L}^{A v e}$. It can be observed in this figure that the highest amount of mismatch is $0.42 \mathrm{MW}$ while the accurate power flow value is equal to $148.9 \mathrm{MW}$. It means that the difference between the linear AC-DC power flow and the linear AC power flow for this line (i.e., line number 32) is just 0.42 MW. This parameter is even smaller for other AC lines in the modified IEEE 57-bus test system. 


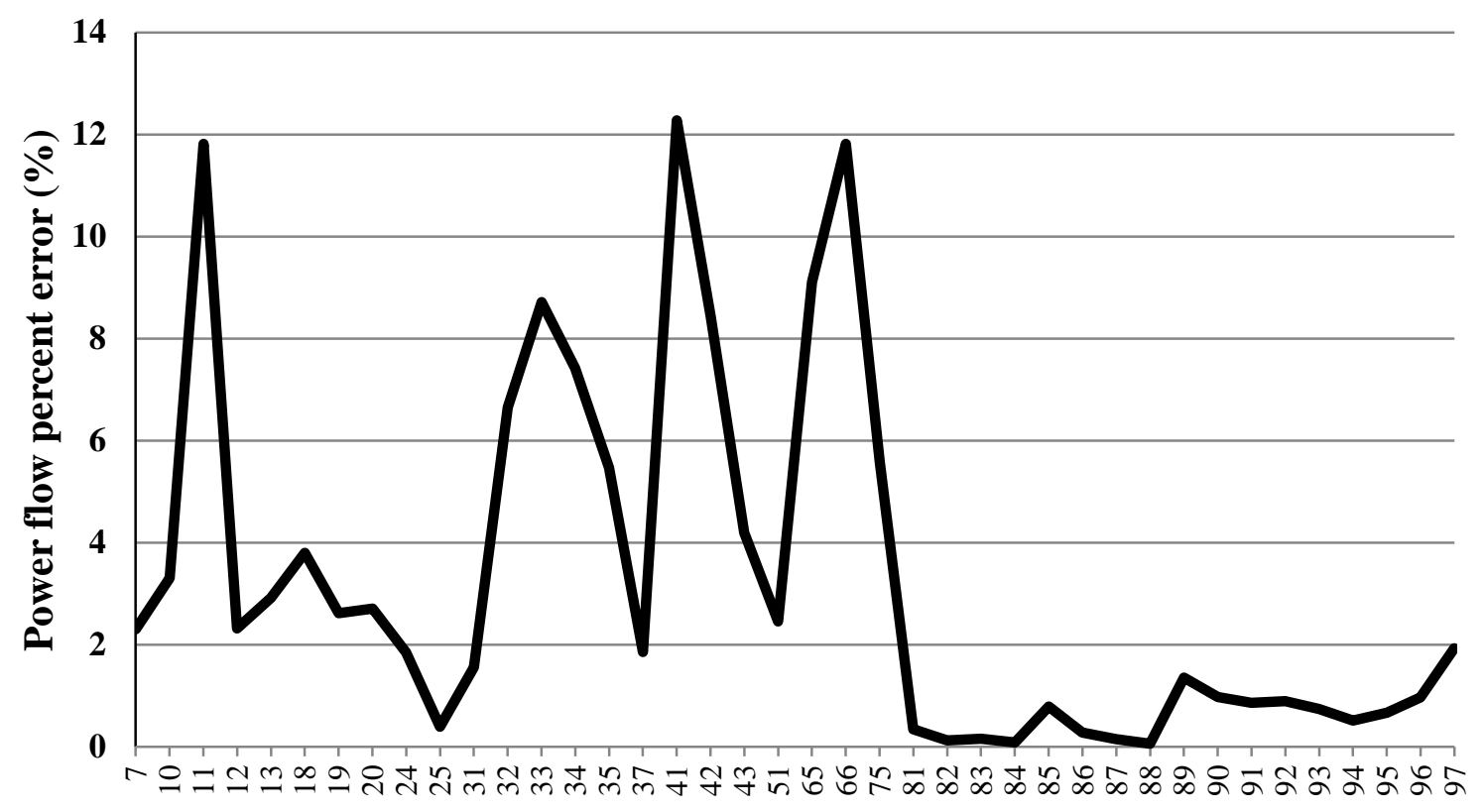

Line number

Figure 3.6: Power flow percent error of the modified IEEE 57-bus test system

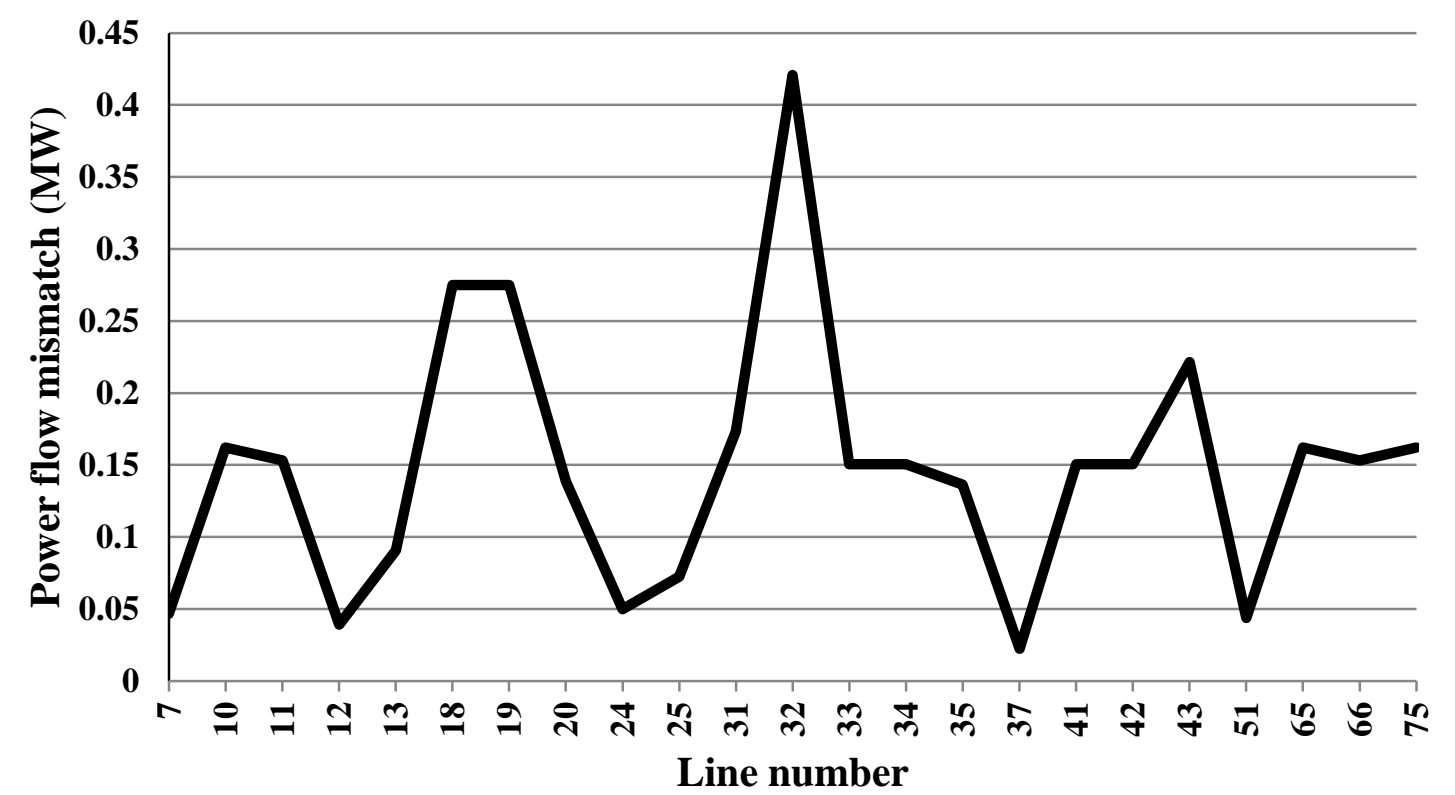

Figure 3.7: Mismatch between linear AC-DC and linear AC power flow values in the modified IEEE 57-bus test system 


\subsection{Modified IEE 118-Bus Test System}

The data of IEEE 118-bus test system is given in [39]. Moreover, the data of the added DC buses and lines are provided in Table A.7. The data of the added AC-DC power converters are tabulated in Table A.8. Based on these two tables, 25 DC lines as well as 4 AC-DC power converters are added to the original IEEE 118-bus test system to prepare it for AC-DC powerflow analysis.

The percent error values of power flow in DC lines and AC-DC power converters of the modified IEEE 118-bus test system are presented in Figure 3.8. The line numbers from 187 to 211 correspond to DC lines and from 212 to 215 correspond to the AC-DC power converters. This figure expresses that no matter of the $P L_{N L}^{A v e}$ value, the percent error between the results of the proposed linear AC-DC and the accurate non-linear AC-DC power flow methods is in an acceptable range.

\subsection{Modified IEE 300-Bus Test System}

IEEE 300-bus test system data can be found in [40]. This network is one of the largest test systems for power flow studies. Like the previous sections, DC buses and lines should be added to the original test system to make an AC-DC network. The data of the added DC buses and DC lines are given in Table A.9 and Table A.10, respectively. Totally, 30 DC lines are added to the original IEEE 300-bus test system. These lines are distributed among 6 DC subgrids connected to the original IEEE 300-bus test system through 6 AC-DC power converters. The data of these AC-DC power converters are shown in Table A.11. As can be seen in this table, 
the voltage transfer ratio $(T)$ values are chosen such that a wide range of voltage magnitude from 0.9516 p.u. to 1.053 p.u. at DC side of the AC-DC power converter is achieved. This variety ensures that the proposed linear AC-DC power-flow analysis method is tested in different conditions including worst cases.

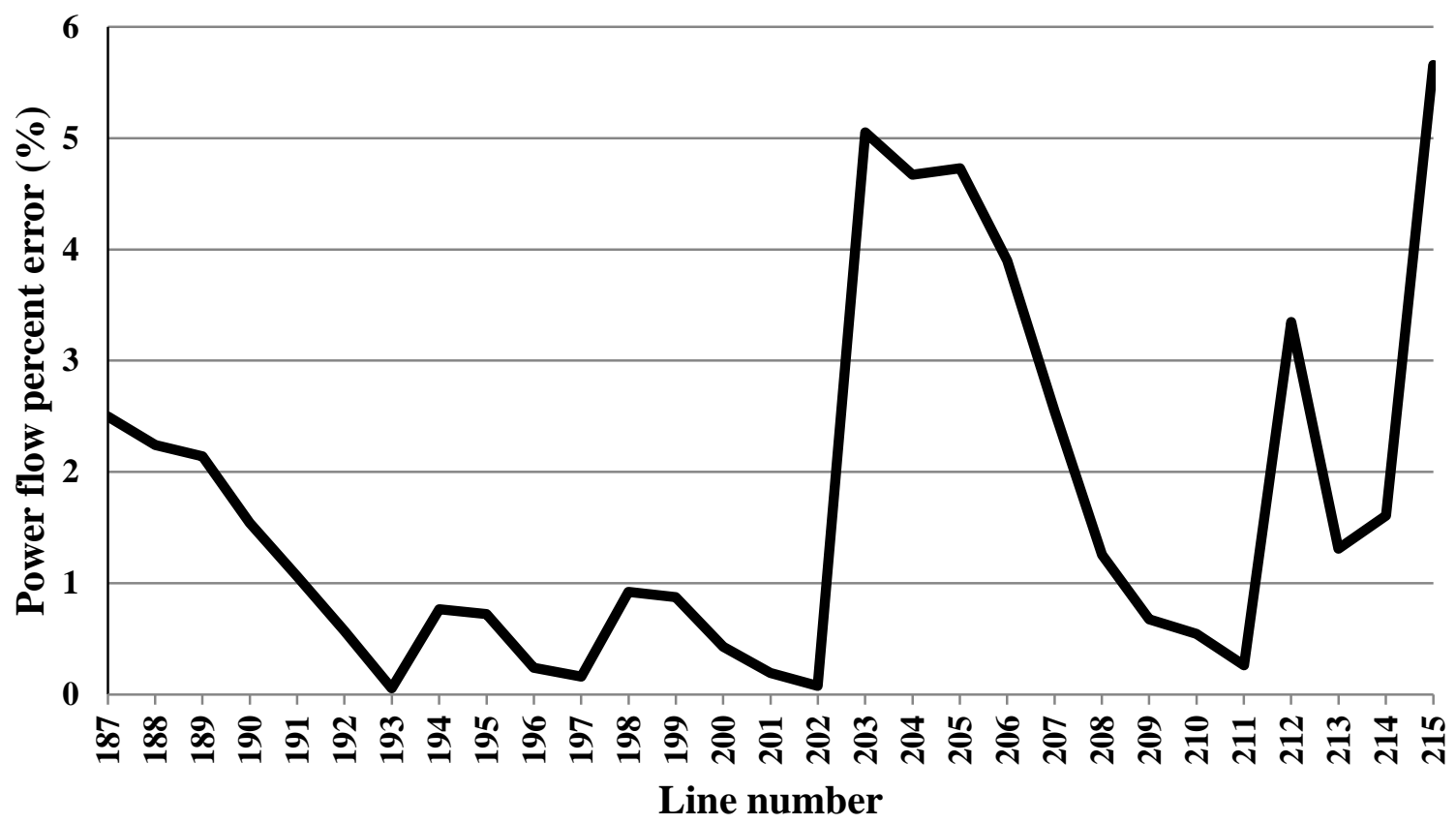

Figure 3.8: Percent error of power flow in DC lines and power converters of the modified IEEE 118-bus test system

The percent error values of power flow in DC lines and AC-DC power converters of the modified IEEE 300-bus test system are presented in Figure 3.9. The line numbers from 412 to 441 correspond to DC lines and from 442 to 447 correspond to the AC-DC power converters. This figure indicates that the percent error values in DC lines are very small and negligible. However, the percent error values of AC-DC power converter are greater but still in a reasonable range. 


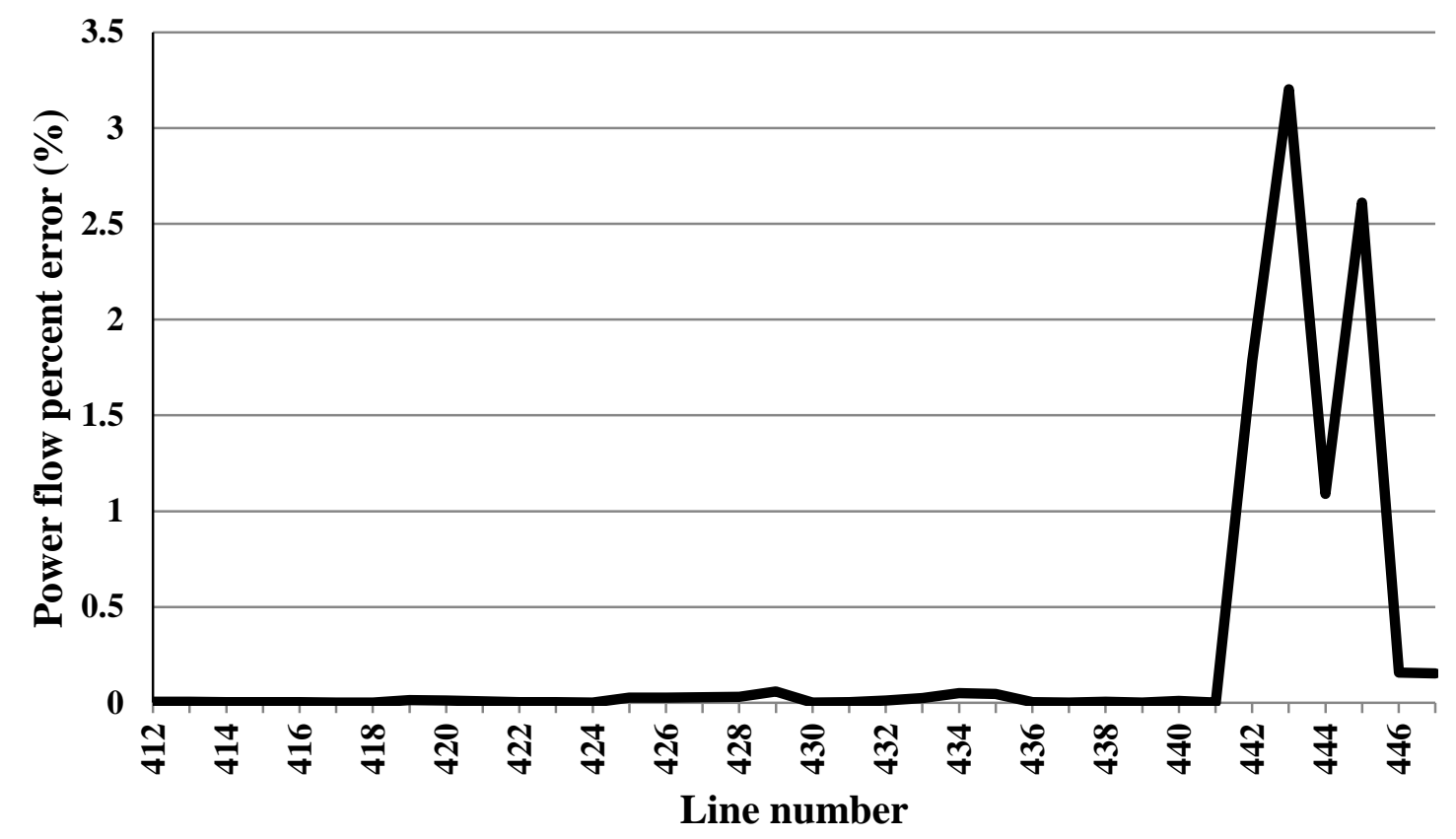

Figure 3.9: Percent error of power flow in DC lines and power converters of the modified IEEE 300-bus test system

The power flow results of the modified test systems are summarized in Table 3.7. The linear and non-linear AC-DC power flow methods are used for the modified test systems while the Newton-Raphson and the linear AC power flow methods are utilized for the original test systems. It should be noted here that only the lines with power flow values more than $P L_{N L}^{A v e}$ are taken into account.

As can be observed, the modified IEEE 300-bus test system has the highest amount of maximum percent error (i.e., 105.36\%) among all seven test systems. However, the small amount of percent error in the second column (i.e., 0.43\%) indicates that the linear AC-DC power flow is very close to linear AC power flow. In other words, the high amount of percent 
error in the modified IEEE 300-bus test system is not caused by the proposed linear AC-DC power-flow analysis method and appears for the same line in linear AC power flow too. This fact can be seen for other maximum percent error values in the first column. It can be also confirmed by the last column of Table 3.7 where the maximum percent error values between the $\mathrm{AC}$ and the proposed linear AC-DC power flows in $\mathrm{AC}$ lines are presented. These values reveal that all linear AC-DC power flow values in AC lines of modified test systems are close to their corresponding linear AC power flow values in original test system. In four test systems, the maximum percent error of linear AC-DC power flow compared to non-linear AC-DC power flow takes place in the line which has the maximum percent error of linear AC-DC power flow compared to linear AC power flow method.

Table 3.7: Summary of the Modified Test Systems

\begin{tabular}{|c|c|c|c|c|}
\hline $\begin{array}{c}\text { No. } \\
\text { of } \\
\text { Buses }\end{array}$ & $\begin{array}{c}\text { Minimum } \\
\text { X/R ratio } \\
\text { of lines }\end{array}$ & $\begin{array}{c}\text { Max percent error - } \\
\text { comparing proposed } \\
\text { linear AC-DC power } \\
\text { flow method with } \\
\text { nonlinear AC-DC Power } \\
\text { Flow method (\%) }\end{array}$ & $\begin{array}{c}\text { Percent Error - } \\
\text { comparing linear } \\
\text { AC power flow } \\
\text { method with } \\
\text { proposed linear AC- } \\
\text { DC power flow } \\
\text { method }(\%)\end{array}$ & $\begin{array}{c}\text { Max percent } \\
\text { error - comparing } \\
\text { linear AC power } \\
\text { flow method with } \\
\text { Newton-Raphson } \\
\text { power flow } \\
\text { method (\%) }\end{array}$ \\
\hline 9 & 4.36 & 13.3 & 0.015 & 0.015 \\
\hline 14 & 0.9 & 6.45 & 0.202 & 0.251 \\
\hline 30 & 0.9 & 7.07 & 0.039 & 0.055 \\
\hline 39 & 1.23 & 7.67 & 0.48 & 0.48 \\
\hline 57 & 0.92 & 12.28 & 0.518 & 0.613 \\
\hline 118 & 2.11 & 48.03 & 0.003 & 0.003 \\
\hline 300 & 0.75 & 105.36 & 0.43 & 0.43 \\
\hline
\end{tabular}

In Table 3.7, the modified IEEE 57-bus test system has the highest percent error values. Apart from the percent error, absolute values of power flow mismatch give a clear 
understanding of the results. The absolute values of mismatch between the proposed linear ACDC and the linear AC power flow methods are demonstrated in Figure 3.7. It can be seen in this figure that the results of the proposed linear AC-DC power flow method are very close to the results of the linear AC power flow method in the original IEEE 57-bus test system. The absolute value of power flow mismatch reaches its maximum value (i.e., $0.42 \mathrm{MW}$ ) in line number 32 .

\subsection{Chapter Summary}

In this chapter, the performance and robustness of the proposed $\mathrm{AC}$-DC power-flow analysis method were investigated. 8 different test systems were utilized for simulation of the proposed method. In the first simulation case and as a numerical example, a 4-bus test system was applied to make the procedure of the proposed method more clear and understandable. Each step of the procedure was described in detail with numerical calculations. The 4-bus test system was implemented in MATLAB/Simulink. Moreover, an accurate conventional nonlinear AC-DC power-flow analysis method s was programmed in MATLAB software. The results of the proposed method were compared with tow aforementioned methods.

In order to comprehensively evaluate the performance of the proposed method in this thesis, seven (7) more test systems of different sizes were used. These original test systems were adapted to AC-DC test system by adding DC lines and AC-DC power converters. The results of all test systems were compared with the non-linear AC-DC power-flow analysis method. Different indices were defined for analyzing the obtained results from the different perspectives. It was shown that the power flow error of AC lines is not caused by the proposed linear AC-DC power-flow analysis method and the error is related to the nature of the original 
AC test system. It also was concluded that the proposed method focuses on DC variables rather than the AC ones. Therefore, it is responsible for the DC variable results.

The sensitivity of the proposed method to AC-DC network load level was tested. It was shown for the modified IEEE 9-bus test system that the proposed method is not sensitive to the load level. The power flow error does not noticeably change even when the load values of all AC and DC buses were doubled. In addition, the sensitivity of the proposed linear AC-DC power-flow analysis method to the voltage magnitude of DC side of the AC-DC power converter was investigated. The voltage transfer ratios of the converters were chosen such that a wide range of the DC voltage magnitude was obtained and the results show that the error values remain small. It was demonstrated that the proposed linear AC-DC power-flow analysis method can be easily integrated into complex planning and operational optimization formulations for AC-DC power systems providing benefits in computation speed. 


\section{CHAPTER 4.}

\section{CONCLUSION}

\subsection{Introduction}

This chapter is devoted to presenting the conclusion of this thesis. A linear power-flow analysis method for AC-DC electric power networks is proposed in this thesis. The thesis includes three main chapters followed by this chapter. The conclusions about the studied topic, its importance, the problem, and the proposed solution are summarized in this chapter. First, a chapter-wise summary is presented describing the contents of the previous chapters. Then, the contribution of this thesis is given. The conclusion of this thesis and the recommendations for future works are the last parts of this chapter. 


\subsection{Summary of the Previous Chapters}

In the first chapter, the topic of the thesis was introduced. The importance of the topic, the industrial applications and academic relevance were explained. It has a wide range of application in power system study from electrical energy market to power system studies and contingency analysis. Moreover, it is a novel method in academic research. The recent related literature was surveyed in the next part. The reviewed literature can be categorized into two groups: non-linear AC-DC power-flow analysis and linear AC power-flow analysis. It was concluded in the first chapter that although different types of non-linear AC-DC power-flow analysis methods have been developed and proposed, to best of the author's knowledge, there is no specific work about the linear AC-DC power-flow analysis. Therefore, the focus of this thesis was to find and proposed a method for linear power-flow analysis in AC-DC electric power networks.

The second chapter of this thesis talked about the problem statement and the proposed solution. It was explained that what exactly the problem is and what we should look for as the solution. In order to have a clear understanding of the related concepts and background, brief reviews of linear power-flow analysis methods for Ac and DC subgrids were presented. The mathematical formulations and the assumptions for obtaining these formulations were described. Then, the general form of linear power-flow equation in AC-DC networks was obtained based on the linear power-flow of AC and DC subgrids in presence of an AC-DC power converter. In the general form, the nodal active power injection vector was related to the unknown variables of AC-DC network by a matrix called $[\boldsymbol{B} \boldsymbol{G}]$ matrix. This matrix can represent the entire AC-DC network. Hence, it was defined as an aim to find the elements of 
the aforementioned matrix. In order to achieve the defined aim, the power flow equations of the AC-DC power converter were linearized. These equations include a parameter the value of which is not known. The linear model of AC-DC power converter was incorporated in a typical AC-DC network and the linear AC-DC power flow equation was yielded. The procedure of the proposed method was also introduced. The procedure explained how to determine the value of $\lambda$ in linearized power flow equations of AC-DC power converter and then how to establish the $[\boldsymbol{B} \boldsymbol{G}]$ matrix.

In the third chapter, to verify the validation of the proposed linear AC-DC power-flow analysis method, it was applied to different test systems. As the first test system, a simple 4-bus AC-DC network was chosen. The detailed explanations and calculations of the proposed method were presented for this test system to clarify the steps of the procedure. Then, other test systems with different sizes and numbers of AC-DC power converter were utilized to test the performance of the proposed methods in different conditions. MATLAB software was used for programming the proposed method as well as a non-linear AC-DC power-flow analysis method. The latter was employed to be compared with the proposed method. The performance of the proposed method was investigated based on different indices and the results were analyzed and the required justifications were presented.

\subsection{Contribution}

In this thesis, as a first contribution, while $\mathrm{AC}$ and $\mathrm{DC}$ lines are represented by susceptance and conductance elements, AC-DC power converters are represented by a proposed linear relationship. The linear model of AC-DC power converter comprises an equivalent reactance in series with an ideal AC-DC converter which accounts for the 
transformation. The phase angle of voltage at the AC side of the ideal AC-DC converter is related to the magnitude of the voltage at DC side of the converter by the parameter $\lambda$.

As a second contribution, a three-step method is proposed linear AC-DC power flow method is proposed. The first step solves the whole network considering it as a linear AC network, yielding bus phase angles at all busses. The second step computes attributes of the proposed linear model of all AC-DC power converters. Here, the procedure for obtaining the parameter $\lambda$ is introduced.

The third step solves the linear model of the AC-DC system including converters, yielding bus phase angles at $\mathrm{AC}$ busses and voltage magnitudes at DC busses. In this third step, the AC sections of full AC-DC power system are represented by real powers and bus phase angles related by respective bus susceptance matrices, the DC sections are represented by real power and bus voltage magnitudes related by respective conductance matrices, and relations between $\mathrm{AC}$ and $\mathrm{DC}$ section busses are related by respective $\lambda$ parameters representing linear models of AC-DC power converters. Aggregating these AC, DC and AC-DC relations, a set of linear power flow equations is presented in a matrix form to represent the entire AC-DC network. This matrix is referred in this paper to as $[B G]$ matrix. Finally, having $[B G]$ matrix and the nodal active power injection vector at both AC and DC busses, the unknown variables of the AC-DC linear power flow analysis, which are phase angles of voltage phasors at AC busses and voltage magnitudes at DC busses, can be calculated. 


\subsection{Conclusion}

While AC-DC power systems have been operating for more than sixty years, absence of linear AC-DC power flow method remains an impediment. Complex optimization tools for planning and operation, which need such a linear AC-DC power flow method, remain challenged.

With rapid use of DC in medium voltage levels, to integrate distributed energy resources such as energy storage, renewables (solar farms), electric vehicles, etc., need for linear AC-DC power flow method for complex AC-DC system optimization is an immediate need. To address this drawback, linear bus-wise power balance equations are developed for AC-DC power systems and presented in this paper.

As a first contribution, while AC and DC lines are represented by susceptance and conductance elements, AC-DC power converters are represented by a proposed linear relationship, which relates $\mathrm{AC}$ bus voltage phase angle to the corresponding DC bus voltage magnitude.

As a second contribution, a three-step linear AC-DC power flow method is proposed. The first step solves the whole network considering it as a linear AC network, yielding bus phase angles at all busses. The second step computes attributes of the proposed linear model of all AC-DC power converters. The third step solves the linear model of the AC-DC system including converters, yielding bus phase angles at AC busses and voltage magnitudes at DC busses.

A numerical example of the proposed linear AC-DC power flow method is presented for a 4-bus test system to clarify the procedure of the method. In this example, comparison is 
drawn with time domain MATLAB/Simulink implementation and conventional accurate nonlinear AC-DC power flow method. The proposed method is demonstrated to be accurate.

Further, the proposed linear AC-DC power flow method is tested with seven (7) standard IEEE test systems, with up to 330 busses. The test results, when compared with accurate conventional nonlinear AC-DC power flow method, demonstrate reliable performance, consistency and reasonable accuracy expected from a linear method.

The method is linear and amenable for future use with complex optimization of AC-DC power systems.

\subsection{Recommendation for Future Work}

In this thesis, a linear AC-DC power-flow analysis method is proposed. However, the proposed methods can be tested and developed for different applications of linear power-flow analysis such as optimal power-flow analysis and contingency analysis of AC-DC electric power networks. 
Table A.1: Data of the added buses and DC lines to the original IEEE 9-bus test system

\begin{tabular}{|c|c|c|c|c|}
\hline Bus No. & Load (MW) & From & To & Resistance (p.u.) \\
\hline 10 & 2.7 & 10 & 11 & 0.017 \\
\hline 11 & 2.6 & 11 & 12 & 0.039 \\
\hline 12 & 2.5 & 12 & 13 & 0.0119 \\
\hline 13 & 2 & 13 & 14 & 0.0085 \\
\hline 14 & 2.6 & 14 & 15 & 0.032 \\
\hline 15 & 2.8 & \multicolumn{3}{|l}{} \\
\cline { 1 - 5 }
\end{tabular}

Table A.2: Data of the added buses and DC lines to the original IEEE 14-bus test system

\begin{tabular}{|c|c|c|c|c|}
\hline Bus No. & Load (MW) & From & To & Resistance (p.u.) \\
\hline 15 & 0.5 & 15 & 16 & 0.09498 \\
\hline 16 & 1 & 16 & 17 & 0.12291 \\
\hline 17 & 1.5 & 17 & 18 & 0.06615 \\
\hline 18 & 2 & 18 & 19 & 0.08205 \\
\hline 19 & 1.2 & 19 & 20 & 0.22092 \\
\hline 20 & 1.5 & 20 & 21 & 0.17093 \\
\hline 21 & 1 & 21 & 22 & 0.03181 \\
\hline 22 & 1.7 & & & \\
\hline
\end{tabular}


Table A.3: Data of the added buses and DC lines to the original IEEE 30-bus test system

\begin{tabular}{|c|c|c|c|c|}
\hline Bus No. & Load (MW) & From & To & Resistance (p.u.) \\
\hline 31 & 1.5 & 31 & 32 & 0.0667 \\
\hline 32 & 2 & 32 & 33 & 0.042 \\
\hline 33 & 2.5 & 33 & 34 & 0.0569 \\
\hline 34 & 2 & 35 & 36 & 0.0692 \\
\hline 35 & 1.5 & 36 & 37 & 0.0767 \\
\hline 36 & 2.5 & 37 & 38 & 0.0872 \\
\hline 37 & 2 & 38 & 39 & 0.097 \\
\hline 38 & 1 & & & \\
\hline 39 & 2 & & & \\
\hline
\end{tabular}

Table A.4: Data of the added buses and DC lines to the original IEEE 39-bus test system

\begin{tabular}{|c|c|c|c|c|}
\hline Bus No. & Load (MW) & From & To & Resistance (p.u.) \\
\hline 40 & 27 & 40 & 41 & 0.0003 \\
\hline 41 & 36 & 41 & 42 & 0.0007 \\
\hline 42 & 18 & 42 & 43 & 0.0013 \\
\hline 43 & 43 & 43 & 44 & 0.0008 \\
\hline 44 & 28 & 44 & 45 & 0.0006 \\
\hline 45 & 30 & 45 & 46 & 0.0022 \\
\hline 46 & 52 & 47 & 48 & 0.0032 \\
\hline 47 & 15 & 48 & 49 & 0.0014 \\
\hline 48 & 19 & 49 & 50 & 0.0043 \\
\hline 49 & 28 & 50 & 51 & 0.0057 \\
\hline 50 & 36 & 51 & 52 & 0.0014 \\
\hline 51 & 20 & 52 & 53 & 0.0013 \\
\hline 52 & 45 & & & \\
\hline 53 & 16 & & & \\
\hline
\end{tabular}


Table A.5: Data of the added buses and DC lines to the original IEEE 57-bus test system

\begin{tabular}{|c|c|c|c|c|}
\hline Bus No. & Load (MW) & From & To & Resistance (p.u.) \\
\hline 58 & 0.5 & 58 & 59 & 0.1 \\
\hline 59 & 0.6 & 59 & 60 & 0.02 \\
\hline 60 & 1 & 60 & 61 & 0.1 \\
\hline 61 & 1.5 & 61 & 62 & 0.135 \\
\hline 62 & 2 & 63 & 64 & 0.207 \\
\hline 63 & 0.6 & 64 & 65 & 0.09 \\
\hline 64 & 0.7 & 65 & 66 & 0.1 \\
\hline 65 & 0.8 & 66 & 67 & 0.1 \\
\hline 66 & 1.1 & 68 & 69 & 0.1 \\
\hline 67 & 1.3 & 69 & 70 & 0.05 \\
\hline 68 & 1.5 & 70 & 71 & 0.05 \\
\hline 69 & 1.2 & 71 & 72 & 0.15 \\
\hline 70 & 1.4 & 72 & 73 & 0.25 \\
\hline 71 & 1.2 & 73 & 74 & 0.5 \\
\hline 72 & 1.1 & & & \\
\hline 73 & 0.8 & & & \\
\hline 74 & 2.5 & & & \\
\hline
\end{tabular}

Table A.6: Data of the added AC-DC power converters to the original IEEE 57-bus test system

\begin{tabular}{|c|c|c|c|c|c|}
\hline 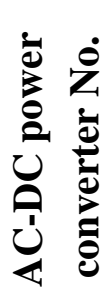 & $\frac{\mathscr{\varrho}}{己}$ & مِ & 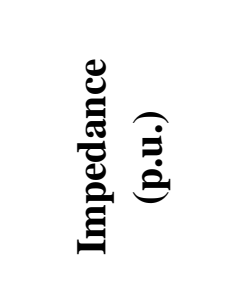 & 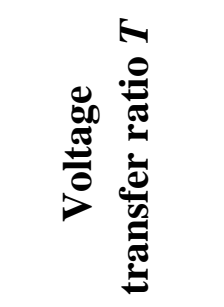 & 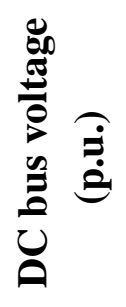 \\
\hline 1 & 19 & 58 & $0.107+0.352 \mathrm{i}$ & $0.915 \measuredangle-13$ & 1.0513 \\
\hline 2 & 30 & 63 & $0.086+0.376 \mathrm{i}$ & $1.01 \measuredangle-18.5$ & 0.9604 \\
\hline 3 & 57 & 68 & $0.113+0.41 \mathrm{i}$ & $0.956 \Varangle-16.5$ & 0.9994 \\
\hline
\end{tabular}


Table A.7: Data of the added buses and DC lines to the original IEEE 118-bus test system

\begin{tabular}{|c|c|c|c|c|}
\hline Bus No. & Load (MW) & From & To & Resistance (p.u.) \\
\hline 119 & 1 & 119 & 120 & 0.0246 \\
\hline 120 & 2 & 120 & 121 & 0.016 \\
\hline 121 & 1.5 & 121 & 122 & 0.0451 \\
\hline 122 & 1.5 & 122 & 123 & 0.0466 \\
\hline 123 & 2 & 123 & 124 & 0.0535 \\
\hline 124 & 2 & 124 & 125 & 0.0605 \\
\hline 125 & 2 & 125 & 126 & 0.00994 \\
\hline 126 & 5 & 127 & 128 & 0.014 \\
\hline 127 & 2 & 128 & 129 & 0.053 \\
\hline 128 & 3 & 129 & 130 & 0.0261 \\
\hline 129 & 5 & 130 & 131 & 0.053 \\
\hline 130 & 7 & 132 & 133 & 0.0105 \\
\hline 131 & 8 & 133 & 134 & 0.03906 \\
\hline 132 & 1.5 & 134 & 135 & 0.0278 \\
\hline 133 & 2.5 & 135 & 136 & 0.022 \\
\hline 134 & 4 & 136 & 137 & 0.0247 \\
\hline 135 & 3 & 138 & 139 & 0.0343 \\
\hline 136 & 7.5 & 139 & 140 & 0.0255 \\
\hline 137 & 8 & 140 & 141 & 0.0503 \\
\hline 138 & 1 & 141 & 142 & 0.0825 \\
\hline 139 & 2 & 142 & 143 & 0.0803 \\
\hline 140 & 2 & 143 & 144 & 0.04739 \\
\hline 141 & 1.5 & 144 & 145 & 0.0317 \\
\hline 142 & 1 & 145 & 146 & 0.0601 \\
\hline 143 & 2.5 & 146 & 147 & 0.0684 \\
\hline 144 & 2 & & & \\
\hline 145 & 3 & & & \\
\hline 146 & 2.5 & & & \\
\hline 147 & 3.5 & & & \\
\hline
\end{tabular}


Table A.8: Data of the added AC-DC power converters to the original IEEE 118-bus test system

\begin{tabular}{|c|c|c|c|c|c|}
\hline 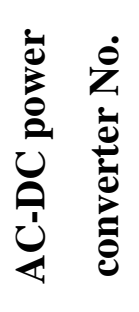 & 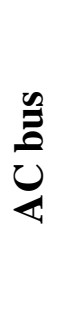 & $\begin{array}{l}\frac{n}{0} \\
\text { Uू }\end{array}$ & 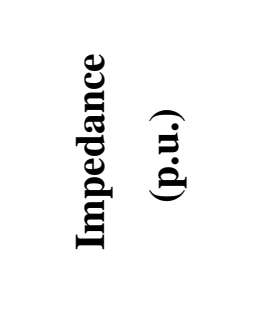 & 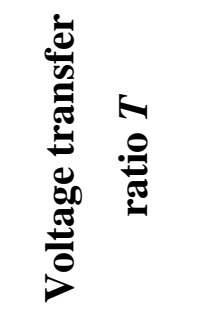 & 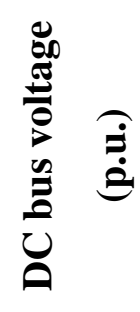 \\
\hline 1 & 33 & 119 & $0.0518+0.188 \mathrm{i}$ & $0.98 \measuredangle-19$ & 0.9906 \\
\hline 2 & 44 & 127 & $0.0393+0.1518 \mathrm{i}$ & $0.99 \measuredangle-16$ & 0.9983 \\
\hline 3 & 52 & 132 & $0.0387+0.1272 \mathrm{i}$ & $0.97 \measuredangle-14$ & 0.9802 \\
\hline 4 & 98 & 138 & $0.0356+0.182 \mathrm{i}$ & $0.979 \measuredangle-2.5$ & 1.0452 \\
\hline
\end{tabular}

Table A.9: Data of the added buses to the original IEEE 300-bus test system

\begin{tabular}{|c|c|c|c|}
\hline Bus No. & Load (MW) & Bus No. & Load (MW) \\
\hline 301 & 5 & 321 & 1.5 \\
\hline 302 & 7 & 325 & 1 \\
\hline 303 & 8 & 326 & 3 \\
\hline 304 & 6 & 327 & 5 \\
\hline 305 & 5.5 & 328 & 6 \\
\hline 306 & 4 & 329 & 6.5 \\
\hline 307 & 6 & 330 & 6 \\
\hline 308 & 3.5 & 331 & 5 \\
\hline 309 & 2 & 332 & 6 \\
\hline 310 & 2.2 & 333 & 5 \\
\hline 311 & 2.2 & 334 & 4.5 \\
\hline 312 & 2 & 335 & 3 \\
\hline 313 & 2.4 & 336 & 0.5 \\
\hline 314 & 2.2 & 337 & 1 \\
\hline 315 & 2.5 & 338 & 1 \\
\hline 316 & 1.5 & 339 & 0.5 \\
\hline 317 & 2 & 340 & 1 \\
\hline 318 & 1 & 341 & 1 \\
\hline
\end{tabular}


Table A.10: Data of the added DC lines to the original IEEE 300-bus test system

\begin{tabular}{|c|c|c|c|c|c|}
\hline From & To & Resistance (p.u.) & From & To & Resistance (p.u.) \\
\hline 301 & 302 & 0.0002 & 318 & 321 & 0.0091 \\
\hline 302 & 303 & 0.0006 & 321 & 325 & 0.00025 \\
\hline 303 & 304 & 0.0002 & 326 & 327 & 0.00065 \\
\hline 304 & 305 & 0.0003 & 327 & 328 & 0.0059 \\
\hline 305 & 306 & 0.00082 & 328 & 329 & 0.0049 \\
\hline 306 & 307 & 0.0002 & 329 & 330 & 0.0059 \\
\hline 307 & 308 & 0.0007 & 330 & 331 & 0.0078 \\
\hline 309 & 310 & 0.00058 & 331 & 332 & 0.0026 \\
\hline 310 & 311 & 0.0011 & 332 & 333 & 0.0076 \\
\hline 311 & 312 & 0.0008 & 333 & 334 & 0.00069 \\
\hline 312 & 313 & 0.0006 & 334 & 335 & 0.000558 \\
\hline 313 & 314 & 0.00076 & 336 & 337 & 0.0019 \\
\hline 314 & 315 & 0.000537 & 337 & 338 & 0.00076 \\
\hline 316 & 317 & 0.0043 & 339 & 340 & 0.0044 \\
\hline 317 & 318 & 0.0039 & 340 & 341 & 0.0002 \\
\hline
\end{tabular}

Table A.11: Data of the added AC-DC power converters to the original IEEE 300-bus test system

\begin{tabular}{|c|c|c|c|c|c|}
\hline 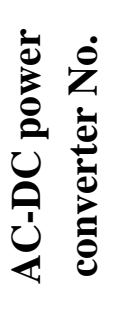 & 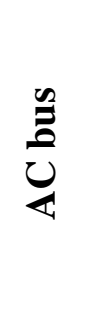 & $\begin{array}{l}\mathscr{U} \\
\frac{0}{0} \\
0\end{array}$ & 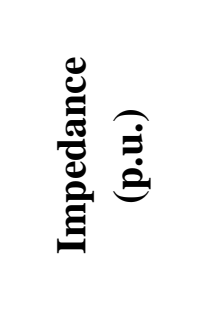 & 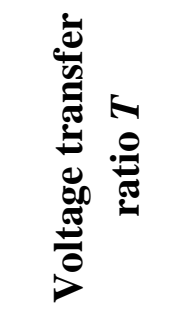 & 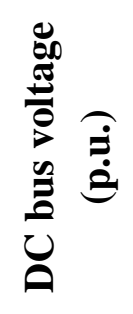 \\
\hline 1 & 52 & 301 & $0.03+0.15 \mathrm{i}$ & 0.987 ð-12 & 1.0377 \\
\hline 2 & 179 & 309 & $0.04+0.17 \mathrm{i}$ & $0.985 \measuredangle-10$ & 0.98 \\
\hline 3 & 208 & 316 & $0.05+0.24 \mathrm{i}$ & 0.987 ð-25 & 1.0209 \\
\hline 4 & 319 & 326 & $0.02+0.1 \mathrm{i}$ & $1.04 \measuredangle 0.7$ & 0.975 \\
\hline 5 & 320 & 336 & $0.06+0.25 \mathrm{i}$ & $0.989 \measuredangle-3$ & 1.053 \\
\hline 6 & 322 & 339 & $0.06+0.3 \mathrm{i}$ & $1.05 \measuredangle-17$ & 0.9516 \\
\hline
\end{tabular}


[1] A. T. Elsayed, A. A. Mohamed, and O. A. Mohammed, "DC microgrids and distribution systems: An overview,” Electr. Power Syst. Res., vol. 119, pp. 407-417, 2015.

[2] A. A. Eajal, M. A. Abdelwahed, E. F. El-Saadany, and K. Ponnambalam, "A Unified Approach to the Power Flow Analysis of AC/DC Hybrid Microgrids," IEEE Trans. Sustain. Energy, vol. 7, no. 3, pp. 1145-1158, 2016.

[3] R. J. Santos et al., "Key energy storage use cases validation under real operational context,” vol. 2017, no. June, pp. 1862-1865, 2017.

[4] V. Verma, L. Goyal, B. Singh, and S. Member, "Converter Based Multi-Terminal DC Sub-Transmission System for City Infeed," Jt. Int. Conf. Power Syst. Technol. IEEE Power India Conf., vol. 00, pp. 1-7, 2008.

[5] L. Wang, Z. H. Yang, X. Y. Lu, and A. V. Prokhorov, "Stability Analysis of a Hybrid 
Multi-Infeed HVdc System Connected Between Two Offshore Wind Farms and Two Power Grids,” IEEE Trans. Ind. Appl., vol. 53, no. 3, pp. 1824-1833, 2017.

[6] E. Unamuno and J. A. Barrena, "Hybrid ac/dc microgrids - Part I: Review and classification of topologies," Renew. Sustain. Energy Rev., vol. 52, pp. 1251-1259, 2015.

[7] E. Unamuno and J. A. Barrena, "Hybrid ac/dc microgrids - Part II: Review and classification of control strategies," Renew. Sustain. Energy Rev., vol. 52, pp. 1123$1134,2015$.

[8] C. Liu, B. Zhang, Y. Hou, and F. F. Wu, "An Improved Approach for AC-DC Power Flow Calculation With Multi-Infeed DC Systems,” vol. 26, no. 2, pp. 862-869, 2011.

[9] J. Beerten, S. Cole, and R. Belmans, "Generalized steady-state Iprotect\{VSC MTDC model for sequential Iprotect\{AC/DC $\}$ power flow algorithms," IEEE Trans. Power Syst., vol. to appear, no. 2, pp. 1-9, 2012.

[10] R. Chai, B. Zhang, J. Dou, Z. Hao, and T. Zheng, "Unified Power Flow Algorithm Based on the NR Method for Hybrid AC/DC Grids Incorporating VSCs," IEEE Trans. Power Syst., vol. 31, no. 6, pp. 4310-4318, 2016.

[11] C. Opathella and B. Venkatesh, "Three-Phase Unbalanced Power Flow Using a-Model of Controllable AC-DC Converters,” vol. 31, no. 6, pp. 4286-4296, 2016.

[12] Y. S. Tzeng, N. Chen, and R. N. Wu, “A Detailed R-L Fed Bridge Converter Model for Power Flow Studies in Industrial AC/DC Power Systems," IEEE Trans. Ind. Electron., vol. 42, no. 5, pp. 531-538, 1995. 
[13] H. M. A. Ahmed, A. B. Eltantawy, and M. M. A. Salama, "A Generalized Approach to the Load Flow Analysis of AC-DC Hybrid Distribution Systems," IEEE Trans. Power Syst., vol. 33, no. 2, pp. 2117-2127, 2018.

[14] X. Zhang, X. Han, M. Yang, D. Sun, Y. Zhang, and W. Gai, "A Novel Power Flow Algorithm for Hybrid AC/DC Power Grids," Electr. Power Components Syst., vol. 45, no. 14, pp. 1-10, 2017.

[15] D. Dhua, S. Huang, and Q. Wu, "Load Flow Analysis of Hybrid AC-DC Power System with Offshore Wind Power,” 2017.

[16] M. Baradar and M. Ghandhari, “A multi-option unified power flow approach for hybrid AC/DC grids incorporating multi-terminal VSC-HVDC," IEEE Trans. Power Syst., vol. 28, no. 3, pp. 2376-2383, 2013.

[17] J. Lei, T. An, Z. Du, and Z. Yuan, “A General Unified AC / DC Power Flow Algorithm with MTDC," IEEE Trans. Power Syst., vol. 32, no. 4, pp. 2837-2846, 2017.

[18] M. Abdi-khorsand, M. Sahraei-ardakani, and Y. M. Al-abdullah, "Corrective Transmission Switching for N- 1-1," IEEE Trans. Power Syst., vol. 32, no. 2, pp. 1606$1615,2017$.

[19] M. Majidi-qadikolai, S. Member, and R. Baldick, "Integration of Contingency Analysis With Systematic Transmission Capacity Expansion Planning : ERCOT Case Study,” vol. 31, no. 3, pp. 1-12, 2015.

[20] K. E. Van Horn, S. Member, A. D. Domínguez-garcía, P. W. Sauer, and L. Fellow, “Measurement-Based Real-Time Security-Constrained Economic Dispatch,” vol. 31, no. 
5, pp. 3548-3560, 2016.

[21] D. A. Tejada-arango, S. Member, S. Pedro, and A. Ramos, "Security Constrained Unit Commitment Using Line Outage Distribution Factors," Renew. Energy Res. Appl., vol. 33, no. 1, pp. 329-337, 2018.

[22] X. Zhang and A. J. Conejo, "Robust Transmission Expansion Planning Representing Long- and Short-Term Uncertainty,” IEEE Trans. Power Syst., vol. 33, no. 2, pp. 13291338, 2018.

[23] K. Purchala, L. Meeus, D. Van Dommelen, and R. Belmans, "Usefulness of DC Power Flow for Active Power Flow Analysis," Power Eng. Soc. Gen. Meet., pp. 454-459, 2005.

[24] A. Garces, "A Linear Three-Phase Load Flow for Power Distribution Systems," IEEE Trans. Power Syst., vol. 31, no. 1, pp. 827-828, 2016.

[25] J. R. Martí, H. Ahmadi, and L. Bashualdo, "Linear Power-Flow Formulation Based on a Voltage-Dependent Load Model," Ieee Trans. Power Deliv., vol. 28, no. 3, p. 9, 2013.

[26] H. Ahmadi, J. R. Marti, and A. Von Meier, "A Linear Power Flow Formulation for Three-Phase Distribution Systems," IEEE Trans. Power Syst., vol. 31, no. 6, pp. 5012$5021,2016$.

[27] Y. Wang, N. Zhang, H. Li, J. Yang, and C. Kang, "Linear three-phase power flow for unbalanced active distribution networks with PV nodes," CSEE J. Power Energy Syst., vol. 3, no. 3, pp. 321-324, 2017. 
[28] T. K. P. Medicherla, M. S. Sachdev, and R. Billinton, "A linear load flow technique for power system reliability studies," Electr. Eng. Journal, Can., vol. 4, no. 3, pp. 17-21, 1979.

[29] A. Lonare, G. Radman, and A. Sekar, "An extended DC-model for estimation of transfer capacity of power systems with phase shifting transformers," North Am. Power Symp. 2010, NAPS 2010, pp. 1-5, 2010.

[30] J. Verboomen, D. Van Hertem, S. Member, P. H. Schavemaker, W. L. Kling, and R. Belmans, "Analytical Approach to Grid Operation With Phase Shifting Transformers," Power, vol. 23, no. 1, pp. 41-46, 2008.

[31] A. P. De Moura, A. A. F. De Moura, D. S. Oliveira, and E. J. Fernandes, "Linear power flow V-theta," Electr. Power Syst. Res., vol. 84, no. 1, pp. 45-57, 2012.

[32] D. Hertem, J. Verboomen, K. Kurchala, R. Belmans, and W. L. L. Kling, "Usefulness of DC power flow for active power flow analysis with flow controlling devices," 8th Int. Conf. AC DC Power Transm., pp. 58-68, 2006.

[33] M. Z. Kamh and R. Iravani, "Steady-state model and power-flow analysis of singlephase electronically coupled distributed energy resources," IEEE Trans. Power Deliv., vol. 27, no. 1, pp. 131-139, 2012.

[34] "IEEE 9-bus modified test system." [Online]. Available: http://www.kios.ucy.ac.cy/testsystems/index.php/dynamic-ieee-test-systems/ieee-9-busmodified-test-system.

"IEEE 14-bus test $\quad$ system." Anline]. Available: 
https://www2.ee.washington.edu/research/pstca/pf14/pg_tca14bus.htm.

"IEEE 30-bus test system." [Online]. Available: https://www2.ee.washington.edu/research/pstca/pf30/pg_tca30bus.htm.

[37] "New England 39-bus test system." [Online]. Available: http://icseg.iti.illinois.edu/ieee39-bus-system/.

"IEEE 57-bus test system." [Online]. Available: https://www2.ee.washington.edu/research/pstca/pf57/pg_tca57bus.htm. https://www2.ee.washington.edu/research/pstca/pf118/pg_tca118bus.htm.

"IEEE 300-bus test system." [Online]. Available:
https://www2.ee.washington.edu/research/pstca/pf300/pg_tca300bus.htm. 